\title{
Assessment of General Toxicity of the Glycyrrhiza New Variety Extract in Rats
}

\author{
Dong-Gu Kim ${ }^{1,+}{ }^{\dagger}$ Jeonghoon Lee ${ }^{2,+}{ }^{\dagger}$, Wonnam Kim ${ }^{3}$, Hyo-Jin An ${ }^{4}$, Jong-Hyun Lee ${ }^{5}$, Jaeki Chang ${ }^{6}$, \\ Sa-Haeng Kang ${ }^{1}$, Young-Jae Song ${ }^{1}$, Yong-Deok Jeon ${ }^{7, *}$ and Jong-Sik Jin ${ }^{1, *(D)}$
}

1 Department of Oriental Medicine Resources, Jeonbuk National University, 79 Gobong-ro, Iksan 54596, Jeollabuk-do, Korea; kdg2409@naver.com (D.-G.K.); kangsh@jbnu.ac.kr (S.-H.K.); dudwoid@naver.com (Y.-J.S.)

2 Herbal Crop Research Division, National Institute of Horticultural and Herbal Science, Rural Development Administration, 92 Bisan-ro, Eumseong 27709, Chungbuk, Korea; artemisia@korea.kr

3 Cnh Center for Cancer Research, 462 Bongeunsa-ro, Gangnam-gu, Seoul 06154, Korea; eb75lab@gmail.com

4 Department of Pharmacology, College of Korean Medicine, Sangji University, 83 Sangjidae-gil, Wonju-si 26339, Gangwon-do, Korea; sangjipharm@gmail.com

5 Department of Pharmacy, College of Pharmacy, Dongduk Women's University, 60 Hwarang-ro 13-gil, Seongbuk-gu, Seoul 02748, Korea; naturalmed@dongduk.ac.kr

6 Crop Production \& Physiology Division, National Institute of Crop Science, Rural Development Administration, 181 Hyeoksin-ro, Wanju 55365, Jeollabuk-do, Korea; changjk@korea.kr

7 Department of Korean Pharmacy, College of Pharmacy, Woosuk University, 443 Samrye-ro, Samrye-eup, Wanju-gun 55338, Jeollabuk-do, Korea

* Correspondence: ydjeon1211jh@woosuk.ac.kr (Y.-D.J.); jongsik.jin@jbnu.ac.kr (J.-S.J.); Tel.: +82-63-290-1503 (Y.-D.J.); +82-63-850-0744 (J.-S.J.)

+ These authors equally contributed to this study.

Citation: Kim, D.-G.; Lee, J.; Kim, W.; An, H.-J.; Lee, J.-H.; Chang, J.; Kang, S.-H.; Song, Y.-J.; Jeon, Y.-D.; Jin, J.-S. Assessment of General Toxicity of the Glycyrrhiza New Variety Extract in Rats. Plants 2021, 10, 1126. https:// doi.org/10.3390/plants10061126

Academic Editors: Yun-Soo Seo and Joong-Sun Kim

Received: 6 May 2021

Accepted: 25 May 2021

Published: 1 June 2021

Publisher's Note: MDPI stays neutral with regard to jurisdictional claims in published maps and institutional affiliations.

Copyright: (c) 2021 by the authors Licensee MDPI, Basel, Switzerland. This article is an open access article distributed under the terms and conditions of the Creative Commons Attribution (CC BY) license (https:/ / creativecommons.org/licenses/by/ $4.0 /)$.
Abstract: The Glycyrrhiza radix (Licorice) is one of the most commonly used medicinal plants in Asian countries, such as China, India, and Korea. It has been traditionally used to treat many diseases, including cough, cold, asthma, fatigue, gastritis, and respiratory tract infections. A Glycyrrhiza new variety, Wongam (WG), has been developed by the Korea Rural Development Administration and revealed pharmacological effects. However, the potential adverse effects of WG have not been revealed yet. This study evaluates the general toxicity of the WG extract through a single and repeated oral dose toxicity study in Sprague-Dawley rats. After single oral dose administration, no significant toxicological changes or mortality was observed up to $5000 \mathrm{mg} / \mathrm{kg}$. Over a 4-week repeated oral dose toxicity study, no adverse effects and target organs were observed up to $5000 \mathrm{mg} / \mathrm{kg} /$ day. Over a 13-week repeated oral dose toxicity study, no mortality or toxicological changes involving ophthalmology, water consumption, or hematology were observed up to $5000 \mathrm{mg} / \mathrm{kg} /$ day. Although other parameters were changed, the alterations in question were not considered toxicologically significant, since responses remained within normal ranges and were not dose-dependent. In conclusion, the no-observed-adverse-effect level (NOAEL) of WG was higher than $5000 \mathrm{mg} / \mathrm{kg} /$ day, and no target organs were identified in rats.

Keywords: Glycyrrhiza; Wongam; single oral dose toxicity study; 4-week repeated oral dose toxicity study; 13-week repeated oral dose toxicity study

\section{Introduction}

Licorice, a perennial plant belonging to the Leguminosae family, is widely distributed in the deserts and on the grasslands of Asia, Europe, and the Americas [1]. It has been used in traditional medicines and folk remedies to treat many diseases, including cough, colds, asthma, fatigue, gastritis, and respiratory tract infections. Furthermore, its current applications extend to the cosmetic and food industries, such as functional foods and food supplements, owing to its numerous positive effects [2]. Various pharmacological properties of licorice have been reported to treat viral infections, inflammation, oxidant 
stress, tumors, asthma, diabetes, depression, allergic responses, and menopausal symptoms [3-7]. Therefore, licorice is listed as a medicinal plant in Korean Pharmacopoeia. Three distinct species of licorice among 22 accepted licorice species are listed in the Korean Pharmacopoeia: G. uralensis Fisch., G. glabra L., and G. inflata Batal. Although licorice is one of the most widely-consumed herbal medicines, with 9000-10,000 tons being consumed annually in South Korea, the domestic self-sufficiency rate is $3-5 \%$, due to low productivity, early leaf fall, and insufficient levels of primary components, such as glycyrrhizin and liquiritigenin, as prescribed in the Korean Pharmacopoeia standards (for glycyrrhizin, a minimum of $2.5 \%$, and for liquiritigenin a minimum of $0.7 \%$ ) [8]. To overcome these problems, a Glycyrrhiza new variety, known as Wongam (WG), has been developed by the Korea Rural Development Administration, which is a hybrid of G. glabra $\times$ G. uralensis. WG has been reported as having higher average yields (227\%) and higher resistance to brown spot disease and lodging than G. uralensis, as well as higher levels of glycyrrhizin $(3.96 \%)$ and liquiritigenin $(0.8 \%)$, in compliance with Korean Pharmacopoeia standards. In addition, WG has been shown as having lower cytotoxicity than G. uralensis in vitro, while its effects variously include enhancing immune response, as well as anti-allergic and anti-neuroinflammatory properties [9-11]. Although several studies have reported the homogeneity and pharmacological effects of WG to register a new species of Glycyrrhiza in Korean Pharmacopoeia, a general toxicity study of WG in rodents has not been conducted to date. Therefore, we evaluated the general toxicity of WG in this study.

The general toxicity test is the most basic and fundamental toxicity test, and is used to identify, evaluate, and determine the safety and the possible risk of adverse effects of substances, such as medicines, cosmetics, health foods, chemicals, and pesticides. It is commonly carried out on animals, such as rats, mice, and dogs, because of scientific, ethical, and regulatory reasons $[12,13]$. The general toxicity test consists of both a single dose and a repeated dose toxicity test, which provide basic toxicity information, including approximate lethal dose (ALD), maximum tolerated dose (MTD), and no-observed-adverseeffect level (NOAEL). The single dose toxicity test provides the most basic information about substances through general symptoms, weight changes, and necropsy findings. The repeated dose toxicity test provides information regarding the long-term effects of substances through general symptoms, weight change, food and water consumption, ophthalmological examination, urinalysis, hematological and blood biochemical tests, necropsy findings, and histopathological examination. Information from the general toxicity test in animals can be used to identify target organ toxicity, to characterize the relationship between drug exposure and response, to determine the likelihood of recovery from drug effects after administration is ended, and to provide information necessary for risk assessment in humans [14,15].

Accordingly, we evaluated the general toxicity of the Glycyrrhiza new variety through a single oral dose and a repeated oral dose toxicity test.

\section{Results}

\subsection{Single Oral Dose Toxicity Study}

Over a 15-day observation period, no mortality was observed in rats of either sex at any of the doses tested. Also, there were no abnormal bodyweight changes in either sex at any doses during the study period (Table 1 ).

No clinical signs related to the administration of WG extract were observed other than compound-colored stool in both sexes of the $2500 \mathrm{mg} / \mathrm{kg}$ group on Day 2, and in the $5000 \mathrm{mg} / \mathrm{kg}$ on Days 1-2 (Table 2). 
Table 1. Bodyweight and mortality in single oral dose toxicity of WG extract.

\begin{tabular}{|c|c|c|c|c|c|c|c|}
\hline \multirow{2}{*}{ Sex } & \multirow{2}{*}{$\begin{array}{c}\text { Dose } \\
\text { (mg/kg) }\end{array}$} & \multicolumn{5}{|c|}{ Bodyweight (g) (Mean \pm SD) } & \multirow{2}{*}{$\begin{array}{c}\text { Mortality } \\
\text { (Dead/Total) }\end{array}$} \\
\hline & & Day 1 & Day 2 & Day 4 & Day 8 & Day 15 & \\
\hline \multirow[t]{4}{*}{ Male } & Control & $281.466 \pm 6.114$ & $314.398 \pm 7.703$ & $331.808 \pm 12.329$ & $362.900 \pm 11.915$ & $410.050 \pm 20.768$ & $0 \%(0 / 5)$ \\
\hline & 1250 & $280.804 \pm 7.599$ & $311.880 \pm 9.621$ & $329.594 \pm 5.731$ & $363.068 \pm 12.778$ & $417.776 \pm 19.317$ & $0 \%(0 / 5)$ \\
\hline & 2500 & $276.854 \pm 6.174$ & $310.744 \pm 7.577$ & $327.868 \pm 10.002$ & $358.008 \pm 14.395$ & $410.658 \pm 27.829$ & $0 \%(0 / 5)$ \\
\hline & 5000 & $279.206 \pm 8.008$ & $312.442 \pm 8.767$ & $330.470 \pm 11.954$ & $362.086 \pm 20.912$ & $409.972 \pm 38.043$ & $0 \%(0 / 5)$ \\
\hline \multirow[t]{4}{*}{ Female } & Control & $197.842 \pm 6.051$ & $220.122 \pm 8.425$ & $227.518 \pm 10.747$ & $234.928 \pm 12.446$ & $257.224 \pm 29.331$ & $0 \%(0 / 5)$ \\
\hline & 1250 & $198.494 \pm 7.890$ & $217.092 \pm 13.885$ & $227.740 \pm 10.210$ & $237.494 \pm 11.940$ & $252.028 \pm 17.321$ & $0 \%(0 / 5)$ \\
\hline & 2500 & $199.578 \pm 4.264$ & $220.060 \pm 7.402$ & $226.420 \pm 7.891$ & $235.222 \pm 7.385$ & $247.224 \pm 12.209$ & $0 \%(0 / 5)$ \\
\hline & 5000 & $196.380 \pm 7.204$ & $214.182 \pm 7.826$ & $225.738 \pm 10.114$ & $238.622 \pm 11.151$ & $248.528 \pm 14.090$ & $0 \%(0 / 5)$ \\
\hline
\end{tabular}

Significant differences were compared with the control group.

Table 2. Clinical signs in single oral dose toxicity of WG extract.

\begin{tabular}{|c|c|c|c|c|c|c|c|c|c|c|c|c|c|c|c|c|c|c|c|c|c|c|}
\hline \multirow{3}{*}{ Sex } & \multirow{3}{*}{$\begin{array}{l}\text { Dose } \\
(\mathrm{mg} / \mathrm{kg})\end{array}$} & \multirow{3}{*}{$\begin{array}{c}\text { Observation } \\
\text { Type }\end{array}$} & \multicolumn{20}{|c|}{ Days Relative to Start Date } \\
\hline & & & 1 & 1 & 1 & 1 & 1 & 1 & 2 & 3 & 4 & 5 & 6 & 7 & 8 & 9 & 10 & 11 & 12 & 13 & 14 & 15 \\
\hline & & & $\begin{array}{c}1 \\
\text { hr }\end{array}$ & $\begin{array}{c}2 \\
\mathrm{hr}\end{array}$ & $\begin{array}{c}3 \\
\mathrm{hr}\end{array}$ & $\begin{array}{c}4 \\
\mathrm{hr}\end{array}$ & $\begin{array}{c}5 \\
\mathrm{hr}\end{array}$ & $\begin{array}{c}6 \\
\mathrm{hr}\end{array}$ & & & & & & & & & & & & & & \\
\hline \multirow[t]{6}{*}{ Male } & Control & Normal & 5 & 5 & 5 & 5 & 5 & 5 & 5 & 5 & 5 & 5 & 5 & 5 & 5 & 5 & 5 & 5 & 5 & 5 & 5 & 5 \\
\hline & 1250 & Normal & 5 & 5 & 5 & 5 & 5 & 5 & 5 & 5 & 5 & 5 & 5 & 5 & 5 & 5 & 5 & 5 & 5 & 5 & 5 & 5 \\
\hline & 2500 & Normal & 5 & 5 & 5 & 5 & 5 & 5 & 0 & 5 & 5 & 5 & 5 & 5 & 5 & 5 & 5 & 5 & 5 & 5 & 5 & 5 \\
\hline & & $\begin{array}{l}\text { Compound- } \\
\text { colored } \\
\text { stool }\end{array}$ & 0 & 0 & 0 & 0 & 0 & 0 & 5 & 0 & 0 & 0 & 0 & 0 & 0 & 0 & 0 & 0 & 0 & 0 & 0 & 0 \\
\hline & 5000 & Normal & 5 & 5 & 5 & 5 & 0 & 0 & 0 & 5 & 5 & 5 & 5 & 5 & 5 & 5 & 5 & 5 & 5 & 5 & 5 & 5 \\
\hline & & $\begin{array}{l}\text { colored } \\
\text { stool }\end{array}$ & 0 & 0 & 0 & 0 & 5 & 5 & 5 & 0 & 0 & 0 & 0 & 0 & 0 & 0 & 0 & 0 & 0 & 0 & 0 & 0 \\
\hline $\begin{array}{l}\text { No. of } \\
\text { animals }\end{array}$ & & & 5 & 5 & 5 & 5 & 5 & 5 & 5 & 5 & 5 & 5 & 5 & 5 & 5 & 5 & 5 & 5 & 5 & 5 & 5 & 5 \\
\hline \multirow[t]{6}{*}{ Female } & Control & Normal & 5 & 5 & 5 & 5 & 5 & 5 & 5 & 5 & 5 & 5 & 5 & 5 & 5 & 5 & 5 & 5 & 5 & 5 & 5 & 5 \\
\hline & 1250 & Normal & 5 & 5 & 5 & 5 & 5 & 5 & 5 & 5 & 5 & 5 & 5 & 5 & 5 & 5 & 5 & 5 & 5 & 5 & 5 & 5 \\
\hline & 2500 & Normal & 5 & 5 & 5 & 5 & 5 & 5 & 0 & 5 & 5 & 5 & 5 & 5 & 5 & 5 & 5 & 5 & 5 & 5 & 5 & 5 \\
\hline & & $\begin{array}{l}\text { Compound- } \\
\text { colored } \\
\text { stool }\end{array}$ & 0 & 0 & 0 & 0 & 0 & 0 & 5 & 0 & 0 & 0 & 0 & 0 & 0 & 0 & 0 & 0 & 0 & 0 & 0 & 0 \\
\hline & 5000 & Normal & 5 & 5 & 5 & 5 & 5 & 0 & 0 & 5 & 5 & 5 & 5 & 5 & 5 & 5 & 5 & 5 & 5 & 5 & 5 & 5 \\
\hline & & $\begin{array}{l}\text { Compound- } \\
\text { colored } \\
\text { stool }\end{array}$ & 0 & 0 & 0 & 0 & 0 & 5 & 5 & 0 & 0 & 0 & 0 & 0 & 0 & 0 & 0 & 0 & 0 & 0 & 0 & 0 \\
\hline $\begin{array}{l}\text { No. of } \\
\text { animals }\end{array}$ & & & 5 & 5 & 5 & 5 & 5 & 5 & 5 & 5 & 5 & 5 & 5 & 5 & 5 & 5 & 5 & 5 & 5 & 5 & 5 & 5 \\
\hline
\end{tabular}

In addition, no gross pathological findings were observed at necropsy in rats of either sex treated with WG extract (Table 3).

\subsection{3-Week Repeated Oral Dose Toxicity Study}

\subsubsection{Mortality, Clinical Signs and Ophthalmological Examination}

No treatment-related mortality was observed in rats of either sex at any doses during the study period. The compound-colored stool was observed in both sexes of the $1250 \mathrm{mg} / \mathrm{kg} /$ day group on Days 7-91, and in the higher dose groups on Days 2-91. Salivation was observed in eight male rats of the $5000 \mathrm{mg} / \mathrm{kg}$ /day group on Days 85-91. Loss of fur and scratched wounds were observed in two male rats of the $5000 \mathrm{mg} / \mathrm{kg} /$ day on Days 44-92 and Days 16-86, respectively. Crust formation was observed in one male rat of the $5000 \mathrm{mg} / \mathrm{kg} /$ day group on Days 17-43. In addition, no treatment-related ophthalmologic abnormalities were observed in rats of either sex at any doses during the study period (Table 4). 


\subsubsection{Bodyweight and Food and Water Consumption}

No significant differences in bodyweight between the control and treatment groups were observed in male rats during the study period. However, a significant increase in bodyweight was observed in female rats of the $2500 \mathrm{mg} / \mathrm{kg}$ / day group in weeks $2,3,5,6$, 7, 8, 10, 11, and 12 (Figure 1).

Table 3. Necropsy findings in single oral dose toxicity of WG extract.

\begin{tabular}{|c|c|c|c|c|c|c|c|c|c|}
\hline \multirow{3}{*}{ Parameters } & \multirow{3}{*}{$\begin{array}{c}\text { Observation } \\
\text { Type }\end{array}$} & \multicolumn{4}{|c|}{ Male } & \multicolumn{4}{|c|}{ Female } \\
\hline & & \multicolumn{4}{|c|}{ Dose (mg/kg) } & \multicolumn{4}{|c|}{ Dose (mg/kg) } \\
\hline & & Control & 1250 & 2500 & 5000 & Control & 1250 & 2500 & 5000 \\
\hline Adrenal Gland & Normal & 5 & 5 & 5 & 5 & 5 & 5 & 5 & 5 \\
\hline Aorta & Normal & 5 & 5 & 5 & 5 & 5 & 5 & 5 & 5 \\
\hline Bone Marrow, Sternum & Normal & 5 & 5 & 5 & 5 & 5 & 5 & 5 & 5 \\
\hline Brain & Normal & 5 & 5 & 5 & 5 & 5 & 5 & 5 & 5 \\
\hline Cervix & Normal & - & - & - & - & 5 & 5 & 5 & 5 \\
\hline Coagulating Gland & Normal & 5 & 5 & 5 & 5 & - & - & - & - \\
\hline Epididymis & Normal & 5 & 5 & 5 & 5 & - & - & - & - \\
\hline Esophagus & Normal & 5 & 5 & 5 & 5 & 5 & 5 & 5 & 5 \\
\hline Eye with Optic Nerve & Normal & 5 & 5 & 5 & 5 & 5 & 5 & 5 & 5 \\
\hline Harderian Gland & Normal & 5 & 5 & 5 & 5 & 5 & 5 & 5 & 5 \\
\hline Heart & Normal & 5 & 5 & 5 & 5 & 5 & 5 & 5 & 5 \\
\hline Cecum & Normal & 5 & 5 & 5 & 5 & 5 & 5 & 5 & 5 \\
\hline Colon & Normal & 5 & 5 & 5 & 5 & 5 & 5 & 5 & 5 \\
\hline Duodenum & Normal & 5 & 5 & 5 & 5 & 5 & 5 & 5 & 5 \\
\hline Ileum & Normal & 5 & 5 & 5 & 5 & 5 & 5 & 5 & 5 \\
\hline Jejunum & Normal & 5 & 5 & 5 & 5 & 5 & 5 & 5 & 5 \\
\hline Rectum & Normal & 5 & 5 & 5 & 5 & 5 & 5 & 5 & 5 \\
\hline Kidney & Normal & 5 & 5 & 5 & 5 & 5 & 5 & 5 & 5 \\
\hline Liver & Normal & 5 & 5 & 5 & 5 & 5 & 5 & 5 & 5 \\
\hline Lung & Normal & 5 & 5 & 5 & 5 & 5 & 5 & 5 & 5 \\
\hline Lymph Node, Mesenteric & Normal & 5 & 5 & 5 & 5 & 5 & 5 & 5 & 5 \\
\hline Lymph Node, Mandibular & Normal & 5 & 5 & 5 & 5 & 5 & 5 & 5 & 5 \\
\hline Skeletal Muscle & Normal & 5 & 5 & 5 & 5 & 5 & 5 & 5 & 5 \\
\hline Nerve, Peripheral & Normal & 5 & 5 & 5 & 5 & 5 & 5 & 5 & 5 \\
\hline Ovary & Normal & - & - & - & - & 5 & 5 & 5 & 5 \\
\hline Pancreas & Normal & 5 & 5 & 5 & 5 & 5 & 5 & 5 & 5 \\
\hline Parathyroid Gland & Normal & 5 & 5 & 5 & 5 & 5 & 5 & 5 & 5 \\
\hline Pituitary Gland & Normal & 5 & 5 & 5 & 5 & 5 & 5 & 5 & 5 \\
\hline Prostate Gland & Normal & 5 & 5 & 5 & 5 & - & - & - & - \\
\hline Salivary Gland & Normal & 5 & 5 & 5 & 5 & 5 & 5 & 5 & 5 \\
\hline Seminal Vesicle & Normal & 5 & 5 & 5 & 5 & - & - & - & - \\
\hline Skin, Mammary & Normal & 5 & 5 & 5 & 5 & 5 & 5 & 5 & 5 \\
\hline Spinal Cord, Thoracic & Normal & 5 & 5 & 5 & 5 & 5 & 5 & 5 & 5 \\
\hline Spleen & Normal & 5 & 5 & 5 & 5 & 5 & 5 & 5 & 5 \\
\hline Stomach & Normal & 5 & 5 & 5 & 5 & 5 & 5 & 5 & 5 \\
\hline Testis & Normal & 5 & 5 & 5 & 5 & - & - & - & - \\
\hline Thymus & Normal & 5 & 5 & 5 & 5 & 5 & 5 & 5 & 5 \\
\hline Thyroid Gland & Normal & 5 & 5 & 5 & 5 & 5 & 5 & 5 & 5 \\
\hline Tongue & Normal & 5 & 5 & 5 & 5 & 5 & 5 & 5 & 5 \\
\hline Trachea & Normal & 5 & 5 & 5 & 5 & 5 & 5 & 5 & 5 \\
\hline Urinary Bladder & Normal & 5 & 5 & 5 & 5 & 5 & 5 & 5 & 5 \\
\hline Uterus & Normal & - & - & - & - & 5 & 5 & 5 & 5 \\
\hline Vagina & Normal & - & - & - & - & 5 & 5 & 5 & 5 \\
\hline Femorotibial Joint & Normal & 5 & 5 & 5 & 5 & 5 & 5 & 5 & 5 \\
\hline No. of animals & & 5 & 5 & 5 & 5 & 5 & 5 & 5 & 5 \\
\hline
\end{tabular}


Table 4. Mortality, clinical signs, and ophthalmological examination in 13 weeks repeated oral dose toxicity of WG extract.

\begin{tabular}{|c|c|c|c|c|c|c|c|c|}
\hline \multirow{4}{*}{ Observation Type } & \multicolumn{8}{|c|}{ From Week 0 to Week 13} \\
\hline & \multicolumn{4}{|c|}{ Male } & \multicolumn{4}{|c|}{ Female } \\
\hline & \multicolumn{4}{|c|}{ Dose (mg/kg/day) } & \multicolumn{4}{|c|}{ Dose (mg/kg/day) } \\
\hline & Control & 1250 & 2500 & 5000 & Control & 1250 & 2500 & 5000 \\
\hline Normal & 10 & 0 & 0 & 0 & 10 & 0 & 0 & 0 \\
\hline Compound-colored stool & 0 & 10 & 10 & 10 & 0 & 10 & 10 & 10 \\
\hline Salivation & 0 & 0 & 0 & 8 & 0 & 0 & 0 & 0 \\
\hline Loss of fur & 0 & 0 & 0 & 2 & 0 & 0 & 0 & 0 \\
\hline Scratched wound & 0 & 0 & 0 & 2 & 0 & 0 & 0 & 0 \\
\hline Crust formation & 0 & 0 & 0 & 1 & 0 & 0 & 0 & 0 \\
\hline Mortality (dead/total) & $0 \%(0 / 10)$ & $0 \%(0 / 10)$ & $0 \%(0 / 10)$ & $0 \%(0 / 10)$ & $0 \%(0 / 10)$ & $0 \%(0 / 10)$ & $0 \%(0 / 10)$ & $0 \%(0 / 10)$ \\
\hline No. of animals & 10 & 10 & 10 & 10 & 10 & 10 & 10 & 10 \\
\hline $\begin{array}{l}\text { Ophthalmological } \\
\text { examination }\end{array}$ & $\mathrm{N}$ & $\mathrm{N}$ & $\mathrm{N}$ & $\mathrm{N}$ & $\mathrm{N}$ & $\mathrm{N}$ & $\mathrm{N}$ & $\mathrm{N}$ \\
\hline No. of animals & 5 & 5 & 5 & 5 & 5 & 5 & 5 & 5 \\
\hline
\end{tabular}

N, Normal.

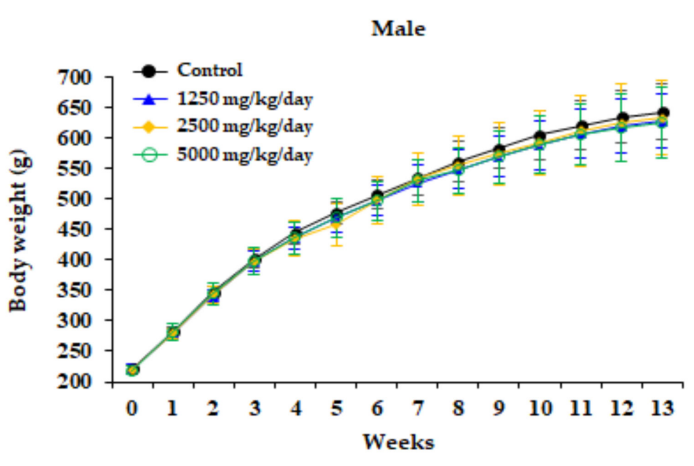

(a)

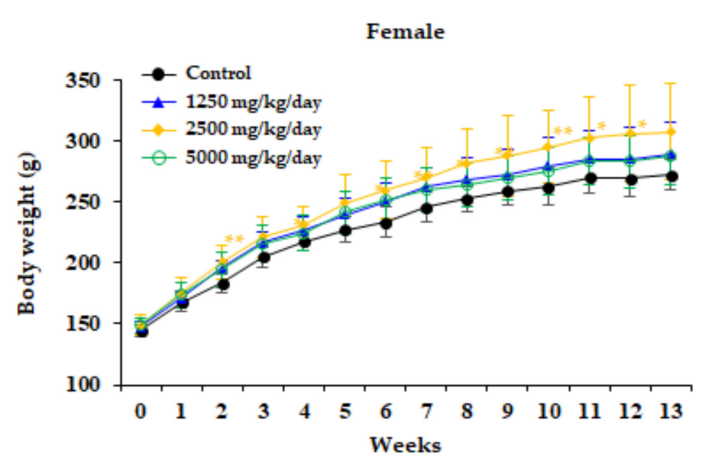

(b)

Figure 1. Bodyweight of male (a) and female (b) rats treated with $0,1250,2500$, and $5000 \mathrm{mg} / \mathrm{kg} /$ day in the 13 weeks repeated oral dose toxicity study of WG extract. The results are presented as the mean \pm standard deviation $(\mathrm{n}=10)$. Significantly different from the control group at ${ }^{*}<0.05,{ }^{* *} P<0.01$.

Food consumption in male rats was not significantly different between the control and treatment groups during the study period. In female rats of the $2500 \mathrm{mg} / \mathrm{kg} /$ day group, however, food consumption increased significantly on Day 21 compared to the control group (Figure 2).

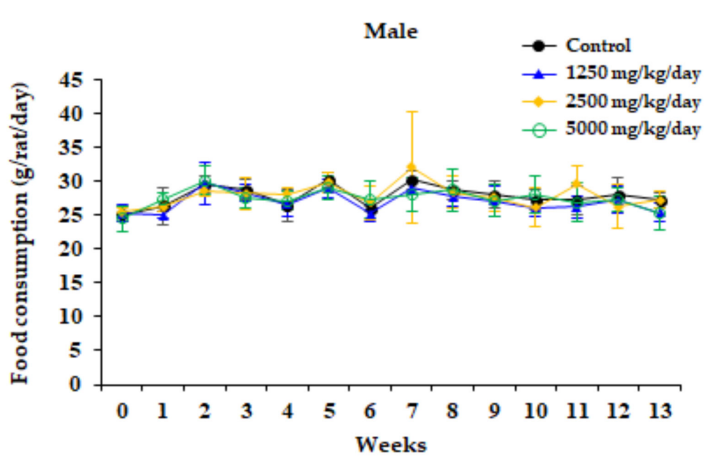

(a)

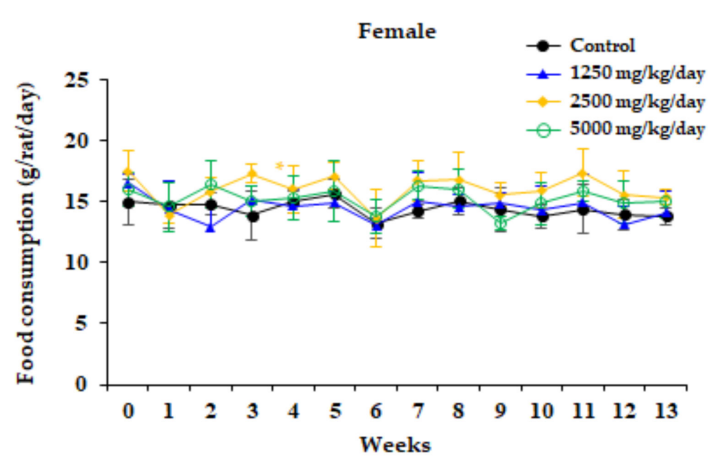

(b)

Figure 2. Food consumption of male (a) and female (b) rats treated with $0,1250,2500$, and $5000 \mathrm{mg} / \mathrm{kg} / \mathrm{day}$ in the 13 weeks repeated oral dose toxicity study of WG extract. Results are presented as the mean \pm standard deviation $(n=10)$. Significantly different from the control group at ${ }^{*} P<0.05$. 
There were no significant changes in water consumption between the control and treatment groups in rats of either sex at any doses during the study period (Figure 3).

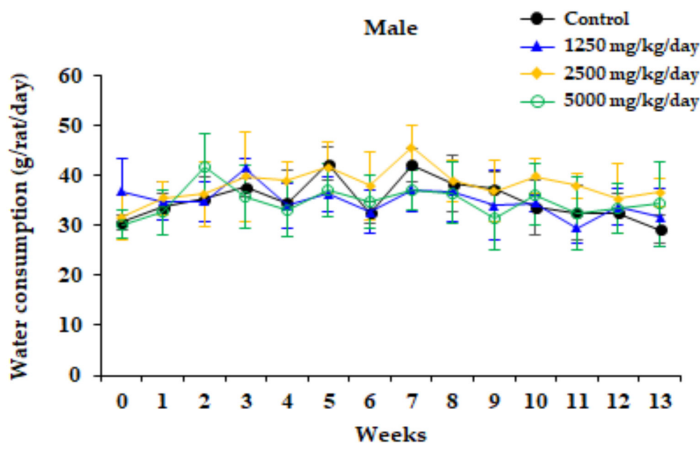

(a)

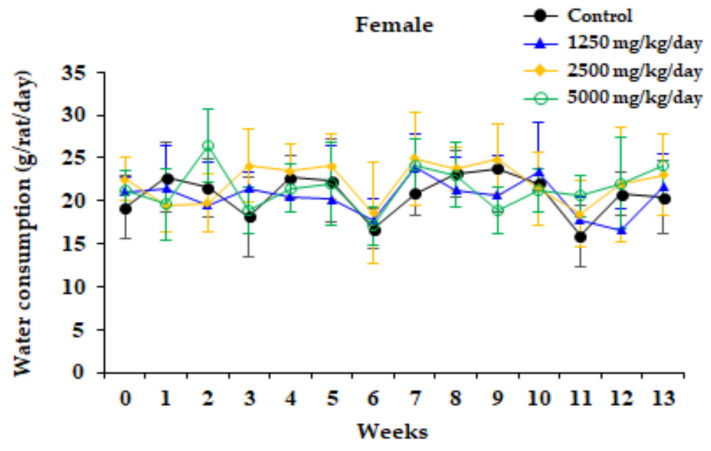

(b)

Figure 3. Water consumption of male (a) and female (b) rats treated with $0,1250,2500$, and $5000 \mathrm{mg} / \mathrm{kg} / \mathrm{day}$ in the 13 weeks repeated oral dose toxicity study of WG extract. Results are presented as the mean \pm standard deviation $(n=10)$.

\subsubsection{Urinalysis}

The results of urinalysis are shown in Table 5 (male rats) and Table 6 (female rats). Ketone body was increased in both sexes of the $5000 \mathrm{mg} / \mathrm{kg} /$ day group. Also, specific gravity was increased in male rats of the 2500 and $5000 \mathrm{mg} / \mathrm{kg} /$ day groups.

Table 5. Urinalysis in 13 weeks repeated oral dose toxicity of WG extract in male rats.

\begin{tabular}{|c|c|c|c|c|c|}
\hline \multirow{2}{*}{ Parameters } & \multirow{2}{*}{ Result } & \multicolumn{4}{|c|}{ Dose (mg/kg/day) } \\
\hline & & Control & 1250 & 2500 & 5000 \\
\hline \multicolumn{6}{|l|}{ Male } \\
\hline Glucose & - & 5 & 5 & 5 & 5 \\
\hline Bilirubin & - & 5 & 5 & 5 & 5 \\
\hline \multirow{5}{*}{ Ketone body } & - & 5 & 5 & 4 & 1 \\
\hline & $+/-$ & 0 & 0 & 0 & 0 \\
\hline & $1+$ & 0 & 0 & 1 & 3 \\
\hline & $2+$ & 0 & 0 & 0 & 1 \\
\hline & $3+$ & 0 & 0 & 0 & 0 \\
\hline \multirow[t]{4}{*}{ Specific gravity } & $\leq 1.005$ & 0 & 0 & 0 & 0 \\
\hline & 1.010 & 5 & 4 & 4 & 1 \\
\hline & 1.015 & 0 & 1 & 1 & 2 \\
\hline & 1.020 & 0 & 0 & 0 & 2 \\
\hline \multirow[t]{4}{*}{$\mathrm{pH}$} & 7.5 & 0 & 0 & 0 & 0 \\
\hline & 8.0 & 2 & 4 & 4 & 0 \\
\hline & 8.5 & 3 & 1 & 1 & 5 \\
\hline & $\geq 9.0$ & 0 & 0 & 0 & 0 \\
\hline \multirow[t]{5}{*}{ Protein } & - & 5 & 3 & 4 & 1 \\
\hline & $+1-$ & 0 & 2 & 1 & 2 \\
\hline & $1+$ & 0 & 0 & 0 & 2 \\
\hline & $2+$ & 0 & 0 & 0 & 0 \\
\hline & $3+$ & 0 & 0 & 0 & 0 \\
\hline \multirow[t]{2}{*}{ Urobilinogen } & 0.2 & 5 & 5 & 5 & 5 \\
\hline & 1.0 & 0 & 0 & 0 & 0 \\
\hline \multirow[t]{2}{*}{ Nitrite } & - & 5 & 5 & 5 & 5 \\
\hline & + & 0 & 0 & 0 & 0 \\
\hline
\end{tabular}


Table 5. Cont.

\begin{tabular}{|c|c|c|c|c|c|}
\hline \multirow{2}{*}{ Parameters } & \multirow{2}{*}{ Result } & \multicolumn{4}{|c|}{ Dose (mg/kg/day) } \\
\hline & & Control & 1250 & 2500 & 5000 \\
\hline \multirow[t]{5}{*}{ Occult blood } & - & 3 & 4 & 4 & 5 \\
\hline & $+/-$ & 2 & 1 & 1 & 0 \\
\hline & $1+$ & 0 & 0 & 0 & 0 \\
\hline & $2+$ & 0 & 0 & 0 & 0 \\
\hline & $3+$ & 0 & 0 & 0 & 0 \\
\hline Clarity & & 5 & 5 & 5 & 5 \\
\hline Color & Yellow & 5 & 5 & 5 & 5 \\
\hline Volume $^{(a)}$ & $\mathrm{mL}$ & $18.80 \pm 8.70$ & $16.00 \pm 3.00$ & $18.60 \pm 3.51$ & $15.00 \pm 4.64$ \\
\hline \multirow[t]{4}{*}{$\mathrm{RBC}$} & - & 4 & 5 & 4 & 5 \\
\hline & $+/-$ & 1 & 0 & 1 & 0 \\
\hline & $1+$ & 0 & 0 & 0 & 0 \\
\hline & $2+$ & 0 & 0 & 0 & 0 \\
\hline \multirow[t]{5}{*}{ WBC } & - & 5 & 5 & 5 & 5 \\
\hline & $+/-$ & 0 & 0 & 0 & 0 \\
\hline & $1+$ & 0 & 0 & 0 & 0 \\
\hline & $2+$ & 0 & 0 & 0 & 0 \\
\hline & $3+$ & 0 & 0 & 0 & 0 \\
\hline \multirow[t]{3}{*}{ Epithelial cell } & - & 5 & 5 & 5 & 5 \\
\hline & $+/-$ & 0 & 0 & 0 & 0 \\
\hline & $1+$ & 0 & 0 & 0 & 0 \\
\hline \multirow[t]{3}{*}{ Casts } & - & 5 & 5 & 5 & 5 \\
\hline & $+1-$ & 0 & 0 & 0 & 0 \\
\hline & $1+$ & 0 & 0 & 0 & 0 \\
\hline No. of animals & & 5 & 5 & 5 & 5 \\
\hline
\end{tabular}

RBC, red blood cell; WBC, white blood cell. ${ }^{(a)}$ Values are mean \pm standard deviation. Significant differences were compared with the control group.

Table 6. Urinalysis in 13 weeks repeated oral dose toxicity of WG extract in female rats.

\begin{tabular}{|c|c|c|c|c|c|}
\hline \multirow{2}{*}{ Parameters } & \multirow{2}{*}{ Result } & \multicolumn{4}{|c|}{ Dose (mg/kg/day) } \\
\hline & & Control & 1250 & 2500 & 5000 \\
\hline \multicolumn{6}{|l|}{ Female } \\
\hline Glucose & - & 5 & 5 & 5 & 5 \\
\hline Bilirubin & - & 5 & 5 & 5 & 5 \\
\hline \multirow{5}{*}{ Ketone body } & - & 5 & 5 & 5 & 0 \\
\hline & $+1-$ & 0 & 0 & 0 & 2 \\
\hline & $1+$ & 0 & 0 & 0 & 3 \\
\hline & $2+$ & 0 & 0 & 0 & 0 \\
\hline & $3+$ & 0 & 0 & 0 & 0 \\
\hline \multirow[t]{4}{*}{ Specific gravity } & $\leq 1.005$ & 0 & 0 & 0 & 0 \\
\hline & 1.010 & 2 & 5 & 4 & 2 \\
\hline & 1.015 & 3 & 0 & 1 & 3 \\
\hline & 1.020 & 0 & 0 & 0 & 0 \\
\hline \multirow[t]{4}{*}{$\mathrm{pH}$} & 7.5 & 0 & 0 & 1 & 0 \\
\hline & 8.0 & 0 & 1 & 0 & 1 \\
\hline & 8.5 & 5 & 4 & 4 & 4 \\
\hline & $\geq 9.0$ & 0 & 0 & 0 & 0 \\
\hline \multirow[t]{5}{*}{ Protein } & - & 5 & 5 & 5 & 4 \\
\hline & $+1-$ & 0 & 0 & 0 & 1 \\
\hline & $1+$ & 0 & 0 & 0 & 0 \\
\hline & $2+$ & 0 & 0 & 0 & 0 \\
\hline & $3+$ & 0 & 0 & 0 & 0 \\
\hline \multirow[t]{2}{*}{ Urobilinogen } & 0.2 & 5 & 5 & 5 & 5 \\
\hline & 1.0 & 0 & 0 & 0 & 0 \\
\hline \multirow[t]{2}{*}{ Nitrite } & - & 5 & 5 & 5 & 5 \\
\hline & + & 0 & 0 & 0 & 0 \\
\hline
\end{tabular}


Table 6. Cont.

\begin{tabular}{|c|c|c|c|c|c|}
\hline \multirow{2}{*}{ Parameters } & \multirow{2}{*}{ Result } & \multicolumn{4}{|c|}{ Dose (mg/kg/day) } \\
\hline & & Control & 1250 & 2500 & 5000 \\
\hline \multirow[t]{5}{*}{ Occult blood } & - & 5 & 5 & 5 & 5 \\
\hline & $+/-$ & 0 & 0 & 0 & 0 \\
\hline & $1+$ & 0 & 0 & 0 & 0 \\
\hline & $2+$ & 0 & 0 & 0 & 0 \\
\hline & $3+$ & 0 & 0 & 0 & 0 \\
\hline Clarity & & 5 & 5 & 5 & 5 \\
\hline Color & Yellow & 5 & 5 & 5 & 5 \\
\hline Volume ${ }^{(a)}$ & $\mathrm{mL}$ & $10.40 \pm 1.67$ & $11.80 \pm 4.44$ & $12.60 \pm 3.85$ & $9.60 \pm 2.30$ \\
\hline \multirow[t]{4}{*}{ RBC } & - & 5 & 5 & 5 & 5 \\
\hline & $+/-$ & 0 & 0 & 0 & 0 \\
\hline & $1+$ & 0 & 0 & 0 & 0 \\
\hline & $2+$ & 0 & 0 & 0 & 0 \\
\hline \multirow[t]{5}{*}{ WBC } & - & 5 & 5 & 5 & 5 \\
\hline & $+/-$ & 0 & 0 & 0 & 0 \\
\hline & $1+$ & 0 & 0 & 0 & 0 \\
\hline & $2+$ & 0 & 0 & 0 & 0 \\
\hline & $3+$ & 0 & 0 & 0 & 0 \\
\hline \multirow[t]{3}{*}{ Epithelial cell } & - & 5 & 5 & 5 & 5 \\
\hline & $+1-$ & 0 & 0 & 0 & 0 \\
\hline & $1+$ & 0 & 0 & 0 & 0 \\
\hline \multirow[t]{3}{*}{ Casts } & - & 5 & 5 & 5 & 5 \\
\hline & $+/-$ & 0 & 0 & 0 & 0 \\
\hline & $1+$ & 0 & 0 & 0 & 0 \\
\hline No. of animals & & 5 & 5 & 5 & 5 \\
\hline
\end{tabular}

$\overline{\mathrm{RBC} \text {, red blood cell; } \mathrm{WBC} \text {, white blood cell. }{ }^{(a)} \text { Values are mean } \pm \text { standard deviation. Significant differences }}$ were compared with the control group.

\subsubsection{Hematology}

Tables 7 and 8 summarize the results of hematological tests involving male and female rats, respectively. No significant differences were observed in any parameters between the control and either of the dose treatment groups rats of either sex.

Table 7. Hematology values in 13 weeks repeated oral dose toxicity of WG extract in male rats.

\begin{tabular}{ccccc}
\hline \multirow{2}{*}{ Parameters } & \multicolumn{4}{c}{ Dose (mg/kg/day) } \\
\cline { 2 - 5 } & Control & $\mathbf{1 2 5 0}$ & $\mathbf{2 5 0 0}$ & $\mathbf{5 0 0 0}$ \\
\hline Male & & & \\
RBC $\left(10^{6} / \mu \mathrm{L}\right)$ & $8.361 \pm 0.390$ & $8.323 \pm 0.278$ & $8.341 \pm 0.401$ & $8.562 \pm 0.295$ \\
$\mathrm{HGB}(\mathrm{g} / \mathrm{dL})$ & $14.940 \pm 0.540$ & $15.03 \pm 0.67$ & $15.21 \pm 0.66$ & $15.20 \pm 0.68$ \\
$\mathrm{HCT}(\%)$ & $44.93 \pm 1.31$ & $45.19 \pm 1.88$ & $45.60 \pm 1.94$ & $45.55 \pm 2.06$ \\
MCV $(\mathrm{fL})$ & $53.82 \pm 1.91$ & $54.31 \pm 1.78$ & $54.74 \pm 0.95$ & $53.22 \pm 1.25$ \\
MCH $(\mathrm{pg})$ & $17.87 \pm 0.76$ & $18.05 \pm 0.53$ & $18.25 \pm 0.29$ & $17.77 \pm 0.48$ \\
$\mathrm{MCHC}(\mathrm{g} / \mathrm{dL})$ & $33.21 \pm 0.56$ & $33.24 \pm 0.47$ & $33.35 \pm 0.35$ & $33.40 \pm 0.46$ \\
RDW $(\%)$ & $13.13 \pm 0.79$ & $13.23 \pm 1.10$ & $13.04 \pm 0.68$ & $13.09 \pm 0.83$ \\
$\mathrm{HDW}(\mathrm{g} / \mathrm{dL})$ & $2.694 \pm 0.196$ & $2.740 \pm 0.295$ & $2.715 \pm 0.276$ & $2.645 \pm 0.249$ \\
RET $(\%)$ & $2.936 \pm 0.945$ & $2.708 \pm 0.623$ & $2.542 \pm 0.591$ & $2.392 \pm 0.324$ \\
PLT $\left(10^{3} / \mu \mathrm{L}\right)$ & $981.7 \pm 134.1$ & $1018.1 \pm 89.6$ & $1032.1 \pm 84.5$ & $1034.8 \pm 88.3$ \\
MPV $(\mathrm{fL})$ & $7.14 \pm 0.35$ & $6.97 \pm 0.52$ & $6.89 \pm 0.47$ & $6.81 \pm 0.57$ \\
WBC $\left(10^{3} / \mu \mathrm{L}\right)$ & $11.081 \pm 2.710$ & $10.770 \pm 1.725$ & $11.430 \pm 2.801$ & $10.761 \pm 2.370$ \\
NEU $(\%)$ & $17.80 \pm 4.52$ & $15.84 \pm 5.35$ & $17.01 \pm 7.46$ & $17.67 \pm 3.24$ \\
NEU $\left(10^{3} / \mu \mathrm{L}\right)$ & $1.923 \pm 0.448$ & $1.705 \pm 0.679$ & $1.965 \pm 1.133$ & $1.893 \pm 0.524$ \\
LYM $(\%)$ & $77.85 \pm 4.97$ & $79.52 \pm 5.69$ & $77.97 \pm 8.03$ & $78.29 \pm 3.78$ \\
\hline
\end{tabular}


Table 7. Cont.

\begin{tabular}{ccccc}
\hline \multirow{2}{*}{ Parameters } & \multicolumn{4}{c}{ Dose $(\mathbf{m g} / \mathbf{k g} /$ day) } \\
\cline { 2 - 5 } & Control & $\mathbf{1 2 5 0}$ & $\mathbf{2 5 0 0}$ & $\mathbf{5 0 0 0}$ \\
\hline LYM $\left(10^{3} / \mu \mathrm{L}\right)$ & $8.680 \pm 2.438$ & $8.565 \pm 1.474$ & $8.894 \pm 2.245$ & $8.437 \pm 1.959$ \\
MONO $(\%)$ & $2.78 \pm 0.67$ & $2.77 \pm 1.01$ & $2.96 \pm 0.98$ & $2.46 \pm 0.58$ \\
MONO $\left(10^{3} / \mu \mathrm{L}\right)$ & $0.301 \pm 0.089$ & $0.295 \pm 0.105$ & $0.341 \pm 0.151$ & $0.263 \pm 0.081$ \\
EOS $(\%)$ & $0.94 \pm 0.31$ & $1.15 \pm 0.38$ & $1.25 \pm 0.41$ & $1.00 \pm 0.41$ \\
EOS $\left(10^{3} / \mu \mathrm{L}\right)$ & $0.103 \pm 0.029$ & $0.122 \pm 0.039$ & $0.136 \pm 0.044$ & $0.105 \pm 0.033$ \\
BASO $(\%)$ & $0.14 \pm 0.07$ & $0.15 \pm 0.07$ & $0.17 \pm 0.07$ & $0.16 \pm 0.05$ \\
BASO $\left(10^{3} / \mu \mathrm{L}\right)$ & $0.018 \pm 0.016$ & $0.017 \pm 0.009$ & $0.018 \pm 0.011$ & $0.016 \pm 0.007$ \\
LUC $(\%)$ & $0.50 \pm 0.14$ & $0.60 \pm 0.30$ & $0.68 \pm 0.36$ & $0.46 \pm 0.12$ \\
LUC $\left(10^{3} / \mu \mathrm{L}\right)$ & $0.058 \pm 0.030$ & $0.065 \pm 0.039$ & $0.075 \pm 0.039$ & $0.048 \pm 0.018$ \\
APTT $(\mathrm{sec})$ & $15.28 \pm 0.62$ & $14.52 \pm 1.98$ & $15.76 \pm 0.84$ & $15.16 \pm 1.05$ \\
PT $(\mathrm{sec})$ & $8.54 \pm 0.21$ & $8.63 \pm 0.38$ & $8.80 \pm 0.21$ & $8.53 \pm 0.22$ \\
No. of animals & 10 & 10 & 10 & 10 \\
\hline
\end{tabular}

RBC, red blood cell; HGB, hemoglobin; HCT, hematocrit; MCV; mean corpuscular volume; $\mathrm{MCH}$, mean corpuscular hemoglobin; MCHC, mean corpuscular hemoglobin concentration; RDW, red cell distribution width; HDW, hemoglobin distribution width; RET, reticulocyte; PLT, platelet; MPV, mean platelet volume; WBC, white blood cell; NEU, neutrophils; LYM, lymphocytes; MONO, monocytes; EOS, eosinophils; BASO, basophils; LUC, large unstained cell, APTT, activated partial thromboplastin time; PT, prothrombin time. Significant differences were compared with the control group.

Table 8. Hematology values in 13 weeks repeated oral dose toxicity of WG extract in female rats.

\begin{tabular}{|c|c|c|c|c|}
\hline \multirow{2}{*}{ Parameters } & \multicolumn{4}{|c|}{ Dose (mg/kg/day) } \\
\hline & Control & 1250 & 2500 & 5000 \\
\hline Female & & & & \\
\hline $\mathrm{RBC}\left(10^{6} / \mu \mathrm{L}\right)$ & $7.788 \pm 0.286$ & $7.587 \pm 0.402$ & $7.347 \pm 0.379$ & $7.570 \pm 0.355$ \\
\hline $\operatorname{HGB}(\mathrm{g} / \mathrm{dL})$ & $14.62 \pm 0.57$ & $14.13 \pm 0.82$ & $14.11 \pm 0.60$ & $14.37 \pm 0.41$ \\
\hline HCT (\%) & $43.08 \pm 1.94$ & $41.82 \pm 2.29$ & $41.94 \pm 1.62$ & $42.43 \pm 1.34$ \\
\hline MCV (fL) & $55.31 \pm 1.00$ & $55.11 \pm 1.19$ & $57.12 \pm 2.25$ & $56.12 \pm 2.04$ \\
\hline MCH (pg) & $18.76 \pm 0.28$ & $18.65 \pm 0.45$ & $19.21 \pm 0.56$ & $19.03 \pm 0.84$ \\
\hline $\mathrm{MCHC}(\mathrm{g} / \mathrm{dL})$ & $33.93 \pm 0.37$ & $33.80 \pm 0.33$ & $33.67 \pm 0.73$ & $33.90 \pm 0.56$ \\
\hline RDW (\%) & $11.26 \pm 0.26$ & $11.30 \pm 0.42$ & $11.65 \pm 0.74$ & $11.45 \pm 0.31$ \\
\hline $\mathrm{HDW}(\mathrm{g} / \mathrm{dL})$ & $2.187 \pm 0.125$ & $2.219 \pm 0.131$ & $2.276 \pm 0.198$ & $2.187 \pm 0.105$ \\
\hline RET (\%) & $2.034 \pm 0.451$ & $2.040 \pm 0.324$ & $2.429 \pm 1.195$ & $2.060 \pm 0.501$ \\
\hline $\operatorname{PLT}\left(10^{3} / \mu \mathrm{L}\right)$ & $1096.8 \pm 110.9$ & $1033.2 \pm 184.2$ & $1111.1 \pm 109.4$ & $1077.0 \pm 52.4$ \\
\hline MPV (fL) & $8.35 \pm 0.36$ & $8.46 \pm 0.55$ & $8.46 \pm 0.19$ & $7.99 \pm 0.80$ \\
\hline $\operatorname{WBC}\left(10^{3} / \mu \mathrm{L}\right)$ & $7.184 \pm 1.303$ & $7.939 \pm 2.870$ & $8.454 \pm 2.965$ & $7.544 \pm 2.327$ \\
\hline NEU $(\%)$ & $10.37 \pm 2.89$ & $14.32 \pm 8.29$ & $11.20 \pm 4.07$ & $10.03 \pm 4.63$ \\
\hline $\operatorname{NEU}\left(10^{3} / \mu \mathrm{L}\right)$ & $0.742 \pm 0.224$ & $1.278 \pm 1.321$ & $0.868 \pm 0.253$ & $0.692 \pm 0.241$ \\
\hline LYM (\%) & $85.68 \pm 3.74$ & $81.68 \pm 8.45$ & $84.80 \pm 4.49$ & $85.01 \pm 4.37$ \\
\hline $\operatorname{LYM}\left(10^{3} / \mu \mathrm{L}\right)$ & $6.164 \pm 1.223$ & $6.340 \pm 1.870$ & $7.250 \pm 2.770$ & $6.474 \pm 2.250$ \\
\hline MONO (\%) & $2.00 \pm 0.57$ & $2.30 \pm 0.77$ & $2.26 \pm 1.12$ & $2.94 \pm 0.47$ \\
\hline $\operatorname{MONO}\left(10^{3} / \mu \mathrm{L}\right)$ & $0.141 \pm 0.036$ & $0.187 \pm 0.102$ & $0.190 \pm 0.107$ & $0.222 \pm 0.066$ \\
\hline EOS $(\%)$ & $1.30 \pm 0.62$ & $1.10 \pm 0.49$ & $0.97 \pm 0.35$ & $1.22 \pm 0.35$ \\
\hline $\operatorname{EOS}\left(10^{3} / \mu \mathrm{L}\right)$ & $0.092 \pm 0.045$ & $0.088 \pm 0.057$ & $0.077 \pm 0.030$ & $0.091 \pm 0.034$ \\
\hline BASO (\%) & $0.14 \pm 0.05$ & $0.14 \pm 0.05$ & $0.16 \pm 0.08$ & $0.14 \pm 0.07$ \\
\hline $\operatorname{BASO}\left(10^{3} / \mu \mathrm{L}\right)$ & $0.010 \pm 0.005$ & $0.010 \pm 0.007$ & $0.015 \pm 0.011$ & $0.011 \pm 0.010$ \\
\hline LUC $(\%)$ & $0.54 \pm 0.27$ & $0.42 \pm 0.15$ & $0.60 \pm 0.18$ & $0.67 \pm 0.39$ \\
\hline $\operatorname{LUC}\left(10^{3} / \mu \mathrm{L}\right)$ & $0.037 \pm 0.016$ & $0.035 \pm 0.018$ & $0.051 \pm 0.029$ & $0.055 \pm 0.055$ \\
\hline APTT (sec) & $13.33 \pm 0.93$ & $14.13 \pm 1.49$ & $14.34 \pm 1.10$ & $13.42 \pm 1.06$ \\
\hline $\mathrm{PT}(\mathrm{sec})$ & $7.63 \pm 0.21$ & $7.69 \pm 0.30$ & $7.58 \pm 0.18$ & $7.58 \pm 0.14$ \\
\hline No. of animals & 10 & 10 & 10 & 10 \\
\hline
\end{tabular}

RBC, red blood cell; HGB, hemoglobin; HCT, hematocrit; MCV; mean corpuscular volume; $\mathrm{MCH}$, mean corpuscular hemoglobin; MCHC, mean corpuscular hemoglobin concentration; RDW, red cell distribution width; HDW, hemoglobin distribution width; RET, reticulocyte; PLT, platelet; MPV, mean platelet volume; WBC, white blood cell; NEU, neutrophils; LYM, lymphocytes; MONO, monocytes; EOS, eosinophils; BASO, basophils; LUC, large unstained cell, APTT, activated partial thromboplastin time; PT, prothrombin time. Significant differences were compared with the control group. 


\subsubsection{Serum Biochemistry}

The results of serum biochemistry are presented in Table 9 (male rats) and Table 10 (female rats). In male rats, Na was significantly increased in the 2500 and $5000 \mathrm{mg} / \mathrm{kg} /$ day groups. In female rats, ALP was significantly increased in the $5000 \mathrm{mg} / \mathrm{kg} /$ day group.

Table 9. Serum biochemical values in 13 weeks repeated oral dose toxicity of WG extract in male rats.

\begin{tabular}{ccccc}
\hline Parameters & \multicolumn{4}{c}{ Dose (mg/kg/day) } \\
\cline { 2 - 5 } & Control & $\mathbf{1 2 5 0}$ & $\mathbf{2 5 0 0}$ & $\mathbf{5 0 0 0}$ \\
Male & & & \\
AST (IU/L) & $94.30 \pm 15.17$ & $96.50 \pm 22.04$ & $98.04 \pm 17.28$ & $102.86 \pm 30.99$ \\
ALT (IU/L) & $26.52 \pm 5.47$ & $28.05 \pm 3.97$ & $27.79 \pm 2.61$ & $29.61 \pm 7.25$ \\
ALP (IU/L) & $81.12 \pm 17.57$ & $85.80 \pm 15.09$ & $75.75 \pm 13.70$ & $80.65 \pm 16.34$ \\
CPK (IU/L) & $373.2 \pm 186.4$ & $352.0 \pm 203.6$ & $319.8 \pm 166.0$ & $369.7 \pm 258.9$ \\
TBIL (mg/dL) & $0.1569 \pm 0.0299$ & $0.1394 \pm 0.0206$ & $0.1540 \pm 0.0308$ & $0.1605 \pm 0.0267$ \\
GLU (mg/dL) & $148.26 \pm 26.75$ & $135.70 \pm 18.55$ & $136.94 \pm 18.61$ & $142.41 \pm 18.87$ \\
TCHO (mg/dL) & $76.0 \pm 20.4$ & $73.8 \pm 20.6$ & $72.5 \pm 25.2$ & $70.1 \pm 19.6$ \\
TG (mg/dL) & $93.2 \pm 62.2$ & $78.7 \pm 25.9$ & $82.2 \pm 35.5$ & $85.2 \pm 21.8$ \\
TP (g/dL) & $6.289 \pm 0.305$ & $6.189 \pm 0.270$ & $6.005 \pm 0.319$ & $6.300 \pm 0.282$ \\
ALB (g/dL) & $2.980 \pm 0.149$ & $2.946 \pm 0.104$ & $2.848 \pm 0.169$ & $2.956 \pm 0.107$ \\
A /G Ratio & $0.901 \pm 0.039$ & $0.911 \pm 0.028$ & $0.903 \pm 0.028$ & $0.886 \pm 0.033$ \\
BUN (mg/dL) & $12.14 \pm 0.78$ & $11.02 \pm 1.30$ & $11.47 \pm 2.10$ & $10.28 \pm 1.52$ \\
CREA (mg/dL) & $0.425 \pm 0.042$ & $0.418 \pm 0.023$ & $0.428 \pm 0.034$ & $0.416 \pm 0.035$ \\
IP (mg/dL) & $5.905 \pm 0.491$ & $5.812 \pm 0.386$ & $5.729 \pm 0.385$ & $5.831 \pm 0.326$ \\
Ca (mg/dL) & $10.171 \pm 0.279$ & $10.178 \pm 0.222$ & $10.015 \pm 0.285$ & $10.139 \pm 0.322$ \\
Na (mmol/L) & $138.526 \pm 0.916$ & $139.004 \pm 0.994$ & $140.314 \pm 1.399 * *$ & $139.969 \pm 0.596 *$ \\
K (mmol/L) & $4.523 \pm 0.341$ & $4.546 \pm 0.178$ & $4.395 \pm 0.278$ & $4.432 \pm 0.234$ \\
Cl (mmol/L) & $100.825 \pm 1.505$ & $101.321 \pm 1.064$ & $101.794 \pm 1.537$ & $100.686 \pm 1.791$ \\
No. of animals & 10 & 10 & 10 & 10 \\
\hline AST aspartate aming & & & \\
\hline
\end{tabular}

AST, aspartate aminotransferase; ALT, alanine aminotransferase; ALP, alkaline phosphatase; CPK, creatine phosphokinase; TBIL, total bilirubin; GLU, glucose; TCHO, total cholesterol; TG, triglyceride; TP, total protein; ALB, albumin; A/G, albumin/globulin; BUN, blood urea nitrogen; CREA, creatinine; IP, inorganic phosphorus $\mathrm{Ca}$, calcium; $\mathrm{Na}$, sodium; $\mathrm{K}$, potassium ion; $\mathrm{Cl}$, chloride ion. Significant differences were compared with the control group, ${ }^{* *} P<0.01$

Table 10. Serum biochemical values in 13 weeks repeated oral dose toxicity of WG extract in female rats.

\begin{tabular}{ccccc}
\hline Parameters & \multicolumn{4}{c}{ Dose $(\mathbf{m g} / \mathbf{k g} /$ day) } \\
\cline { 2 - 5 } & Control & $\mathbf{1 2 5 0}$ & $\mathbf{2 5 0 0}$ & $\mathbf{5 0 0 0}$ \\
\hline Female & & & & \\
AST (IU/L) & $92.39 \pm 40.42$ & $121.73 \pm 98.49$ & $97.90 \pm 38.84$ & $92.33 \pm 23.21$ \\
ALT (IU/L) & $26.07 \pm 6.72$ & $36.11 \pm 39.39$ & $27.88 \pm 11.12$ & $24.07 \pm 4.37$ \\
ALP (IU/L) & $33.90 \pm 8.57$ & $37.48 \pm 9.41$ & $38.96 \pm 14.05$ & $47.66 \pm 10.38 *$ \\
CPK (IU/L) & $234.1 \pm 170.5$ & $237.0 \pm 125.5$ & $247.6 \pm 134.9$ & $251.3 \pm 153.6$ \\
TBIL (mg/dL) & $0.2024 \pm 0.0181$ & $0.2083 \pm 0.0272$ & $0.2166 \pm 0.0577$ & $0.1884 \pm 0.0252$ \\
GLU (mg/dL) & $120.23 \pm 13.89$ & $120.25 \pm 7.89$ & $130.79 \pm 15.62$ & $126.40 \pm 18.62$ \\
TCHO (mg/dL) & $80.1 \pm 15.5$ & $88.9 \pm 31.6$ & $78.1 \pm 20.4$ & $72.3 \pm 16.8$ \\
TG (mg/dL) & $31.4 \pm 7.6$ & $35.2 \pm 7.1$ & $37.3 \pm 8.2$ & $37.0 \pm 13.1$ \\
TP (g/dL) & $6.643 \pm 0.404$ & $6.736 \pm 0.498$ & $6.813 \pm 0.376$ & $6.584 \pm 0.303$ \\
ALB (g/dL) & $3.518 \pm 0.260$ & $3.498 \pm 0.317$ & $3.536 \pm 0.264$ & $3.402 \pm 0.211$ \\
A/G Ratio & $1.127 \pm 0.055$ & $1.079 \pm 0.048$ & $1.079 \pm 0.066$ & $1.069 \pm 0.056$ \\
BUN (mg/dL) & $15.16 \pm 2.75$ & $13.52 \pm 2.61$ & $14.15 \pm 2.71$ & $14.85 \pm 1.92$ \\
CREA (mg/dL) & $0.497 \pm 0.054$ & $0.509 \pm 0.041$ & $0.552 \pm 0.081$ & $0.538 \pm 0.057$ \\
IP (mg/dL) & $5.545 \pm 0.440$ & $5.616 \pm 0.446$ & $5.355 \pm 0.332$ & $5.427 \pm 0.290$ \\
Ca (mg/dL) & $10.422 \pm 0.340$ & $10.479 \pm 0.331$ & $10.302 \pm 0.428$ & $10.236 \pm 0.297$ \\
Na (mmol/L) & $136.228 \pm 1.378$ & $136.250 \pm 1.150$ & $136.242 \pm 0.942$ & $137.330 \pm 0.949$ \\
K (mmol/L) & $3.850 \pm 0.341$ & $3.995 \pm 0.183$ & $3.779 \pm 0.222$ & $3.763 \pm 0.219$ \\
Cl (mmol/L) & $100.936 \pm 1.173$ & $100.298 \pm 0.764$ & $100.117 \pm 1.849$ & $100.866 \pm 1.598$ \\
No. of animals & 10 & 10 & 10 & 10 \\
\hline AST aspartate aminotrang & & & \\
\hline
\end{tabular}

AST, aspartate aminotransferase; ALT, alanine aminotransferase; ALP, alkaline phosphatase; CPK, creatine phosphokinase; TBIL, total bilirubin; GLU, glucose; TCHO, total cholesterol; TG, triglyceride; TP, total protein; ALB, albumin; A/G, albumin/globulin; BUN, blood urea nitrogen; CREA, creatinine; IP, inorganic phosphorus; $\mathrm{Ca}$, calcium; $\mathrm{Na}$, sodium; $\mathrm{K}$, potassium ion; $\mathrm{Cl}$, chloride ion. Significant differences were compared with the control group, ${ }^{*} P<0.05$. 


\subsubsection{Absolute and Relative Organ Weight}

The results of absolute and relative organ weight in male and female rats are shown in Tables 11 and 12, respectively. In male rats, the relative organ weight of the left kidney in the $5000 \mathrm{mg} / \mathrm{kg} /$ day group and the absolute organ weight of the right testis in the $1250 \mathrm{mg} / \mathrm{kg} /$ day group were significantly increased. In female rats, the absolute organ weight of the lung was increased in the 1250 and $2500 \mathrm{mg} / \mathrm{kg} /$ day groups. Additionally, bodyweights were significantly increased in female rats of the $2500 \mathrm{mg} / \mathrm{kg} /$ day group.

Table 11. Absolute and relative organ weights in 13 weeks repeated oral dose toxicity of WG extract in male rats.

\begin{tabular}{|c|c|c|c|c|}
\hline \multirow{2}{*}{ Parameters } & \multicolumn{4}{|c|}{ Dose (mg/kg/day) } \\
\hline & Control & 1250 & 2500 & 5000 \\
\hline Male & & & & \\
\hline Bodyweights (g) ${ }^{\text {(a) }}$ & $607.806 \pm 46.392$ & $594.079 \pm 41.628$ & $598.491 \pm 58.535$ & $591.249 \pm 55.237$ \\
\hline Brain $(\mathrm{g})$ & $2.230 \pm 0.100$ & $2.194 \pm 0.073$ & $2.219 \pm 0.093$ & $2.227 \pm 0.117$ \\
\hline$\%$ to bodyweight & $0.368 \pm 0.023$ & $0.370 \pm 0.020$ & $0.374 \pm 0.036$ & $0.378 \pm 0.022$ \\
\hline Pituitary Gland & $0.015 \pm 0.003$ & $0.014 \pm 0.003$ & $0.015 \pm 0.003$ & $0.014 \pm 0.002$ \\
\hline$\%$ to bodyweight & $0.002 \pm 0.000$ & $0.002 \pm 0.000$ & $0.003 \pm 0.001$ & $0.002 \pm 0.000$ \\
\hline Lung & $1.888 \pm 0.130$ & $1.903 \pm 0.175$ & $1.902 \pm 0.088$ & $1.953 \pm 0.275$ \\
\hline$\%$ to bodyweight & $0.311 \pm 0.019$ & $0.321 \pm 0.028$ & $0.321 \pm 0.037$ & $0.330 \pm 0.029$ \\
\hline Heart & $1.704 \pm 0.102$ & $1.661 \pm 0.175$ & $1.664 \pm 0.101$ & $1.757 \pm 0.213$ \\
\hline$\%$ to bodyweight & $0.281 \pm 0.016$ & $0.280 \pm 0.023$ & $0.280 \pm 0.024$ & $0.297 \pm 0.025$ \\
\hline Thymus & $0.400 \pm 0.095$ & $0.379 \pm 0.078$ & $0.315 \pm 0.066$ & $0.381 \pm 0.102$ \\
\hline$\%$ to bodyweight & $0.066 \pm 0.015$ & $0.064 \pm 0.012$ & $0.053 \pm 0.009$ & $0.064 \pm 0.013$ \\
\hline Spleen & $1.018 \pm 0.165$ & $0.929 \pm 0.207$ & $1.039 \pm 0.196$ & $0.965 \pm 0.181$ \\
\hline$\%$ to bodyweight & $0.168 \pm 0.029$ & $0.155 \pm 0.026$ & $0.175 \pm 0.034$ & $0.163 \pm 0.021$ \\
\hline Adrenal (left) & $0.033 \pm 0.006$ & $0.032 \pm 0.007$ & $0.032 \pm 0.003$ & $0.030 \pm 0.006$ \\
\hline$\%$ to bodyweight & $0.005 \pm 0.001$ & $0.005 \pm 0.001$ & $0.005 \pm 0.001$ & $0.005 \pm 0.001$ \\
\hline Adrenal (right) & $0.030 \pm 0.007$ & $0.031 \pm 0.005$ & $0.030 \pm 0.003$ & $0.029 \pm 0.007$ \\
\hline$\%$ to bodyweight & $0.005 \pm 0.001$ & $0.005 \pm 0.001$ & $0.005 \pm 0.001$ & $0.005 \pm 0.001$ \\
\hline Kidney (left) & $1.707 \pm 0.187$ & $1.699 \pm 0.169$ & $1.732 \pm 0.183$ & $1.869 \pm 0.232$ \\
\hline$\%$ to bodyweight & $0.282 \pm 0.031$ & $0.286 \pm 0.020$ & $0.291 \pm 0.028$ & $0.316 \pm 0.032 *$ \\
\hline Kidney (right) & $1.722 \pm 0.188$ & $1.703 \pm 0.137$ & $1.705 \pm 0.206$ & $1.839 \pm 0.186$ \\
\hline$\%$ to bodyweight & $0.284 \pm 0.032$ & $0.287 \pm 0.017$ & $0.286 \pm 0.030$ & $0.311 \pm 0.024$ \\
\hline Liver & $15.778 \pm 2.144$ & $14.926 \pm 1.693$ & $14.962 \pm 2.000$ & $15.924 \pm 1.892$ \\
\hline$\%$ to bodyweight & $2.595 \pm 0.291$ & $2.507 \pm 0.146$ & $2.499 \pm 0.198$ & $2.693 \pm 0.189$ \\
\hline Testis (left) & $1.894 \pm 0.107$ & $2.046 \pm 0.216$ & $1.880 \pm 0.065$ & $1.984 \pm 0.182$ \\
\hline$\%$ to bodyweight & $0.313 \pm 0.031$ & $0.345 \pm 0.034$ & $0.317 \pm 0.033$ & $0.338 \pm 0.042$ \\
\hline Testis (right) & $1.885 \pm 0.109$ & $2.053 \pm 0.198 *$ & $1.884 \pm 0.096$ & $1.976 \pm 0.176$ \\
\hline$\%$ to bodyweight & $0.312 \pm 0.031$ & $0.346 \pm 0.033$ & $0.318 \pm 0.038$ & $0.336 \pm 0.041$ \\
\hline Epididymis (left) & $0.773 \pm 0.067$ & $0.760 \pm 0.079$ & $0.781 \pm 0.069$ & $0.784 \pm 0.049$ \\
\hline \% to bodyweight & $0.128 \pm 0.011$ & $0.128 \pm 0.010$ & $0.131 \pm 0.008$ & $0.133 \pm 0.011$ \\
\hline Epididymis (right) & $0.772 \pm 0.051$ & $0.782 \pm 0.061$ & $0.789 \pm 0.070$ & $0.825 \pm 0.071$ \\
\hline$\%$ to bodyweight & $0.128 \pm 0.012$ & $0.132 \pm 0.011$ & $0.132 \pm 0.010$ & $0.140 \pm 0.009$ \\
\hline Prostate Gland & $0.622 \pm 0.134$ & $0.680 \pm 0.200$ & $0.725 \pm 0.188$ & $0.760 \pm 0.181$ \\
\hline$\%$ to bodyweight & $0.102 \pm 0.019$ & $0.115 \pm 0.035$ & $0.121 \pm 0.028$ & $0.129 \pm 0.031$ \\
\hline No. of animals & 10 & 10 & 10 & 10 \\
\hline
\end{tabular}

(a) Bodyweights were measured immediately prior to necropsy after an overnight fast. Significant differences were compared with the control group.

\subsubsection{Necropsy Findings}

Tables 13 and 14 summarize the results of necropsy findings in male and female rats, respectively. In one male rat of the control group, the absence of the left parathyroid gland and the left thyroid gland, and enlargement of the right thyroid gland were observed. In one male rat of the $5000 \mathrm{mg} / \mathrm{kg} /$ day group, alopecia and skin crusting were observed. Retention of clear fluid in the uterus was observed in one female rat in the control group, three female rats of the $1250 \mathrm{mg} / \mathrm{kg}$ / day group, two female rats of the $2500 \mathrm{mg} / \mathrm{kg} /$ day group, and one female rat of the $5000 \mathrm{mg} / \mathrm{kg} /$ day group. In addition, discoloration of the liver was observed in one female rat of the $1250 \mathrm{mg} / \mathrm{kg} /$ day group. 
Table 12. Absolute and relative organ weights in 13 weeks repeated oral dose toxicity of WG extract in female rats.

\begin{tabular}{|c|c|c|c|c|}
\hline \multirow{2}{*}{ Parameters } & \multicolumn{4}{|c|}{ Dose (mg/kg/day) } \\
\hline & Control & 1250 & 2500 & 5000 \\
\hline \multicolumn{5}{|l|}{ Female } \\
\hline Bodyweights (g) (a) & $257.329 \pm 12.846$ & $273.903 \pm 24.745$ & $292.277 \pm 37.093 *$ & $271.045 \pm 20.724$ \\
\hline Brain & $1.957 \pm 0.093$ & $2.030 \pm 0.078$ & $2.017 \pm 0.048$ & $2.023 \pm 0.093$ \\
\hline$\%$ to bodyweight & $0.763 \pm 0.062$ & $0.746 \pm 0.072$ & $0.699 \pm 0.080$ & $0.751 \pm 0.076$ \\
\hline Pituitary & $0.018 \pm 0.003$ & $0.019 \pm 0.003$ & $0.021 \pm 0.005$ & $0.019 \pm 0.003$ \\
\hline$\%$ to bodyweight & $0.007 \pm 0.001$ & $0.007 \pm 0.001$ & $0.007 \pm 0.002$ & $0.007 \pm 0.001$ \\
\hline Lung & $1.121 \pm 0.154$ & $1.245 \pm 0.067 *$ & $1.274 \pm 0.114 *$ & $1.225 \pm 0.086$ \\
\hline$\%$ to bodyweight & $0.436 \pm 0.059$ & $0.458 \pm 0.046$ & $0.439 \pm 0.046$ & $0.454 \pm 0.046$ \\
\hline Heart & $0.981 \pm 0.157$ & $0.958 \pm 0.088$ & $0.982 \pm 0.133$ & $0.933 \pm 0.088$ \\
\hline$\%$ to bodyweight & $0.382 \pm 0.066$ & $0.351 \pm 0.029$ & $0.336 \pm 0.023$ & $0.344 \pm 0.021$ \\
\hline Thymus & $0.267 \pm 0.044$ & $0.262 \pm 0.064$ & $0.274 \pm 0.072$ & $0.271 \pm 0.053$ \\
\hline$\%$ to bodyweight & $0.104 \pm 0.021$ & $0.095 \pm 0.020$ & $0.094 \pm 0.021$ & $0.100 \pm 0.016$ \\
\hline Spleen & $0.454 \pm 0.046$ & $0.481 \pm 0.065$ & $0.527 \pm 0.152$ & $0.493 \pm 0.074$ \\
\hline$\%$ to bodyweight & $0.177 \pm 0.019$ & $0.176 \pm 0.025$ & $0.180 \pm 0.043$ & $0.183 \pm 0.032$ \\
\hline Adrenal (left) & $0.032 \pm 0.005$ & $0.035 \pm 0.007$ & $0.035 \pm 0.006$ & $0.036 \pm 0.007$ \\
\hline$\%$ to bodyweight & $0.012 \pm 0.002$ & $0.013 \pm 0.002$ & $0.012 \pm 0.002$ & $0.013 \pm 0.003$ \\
\hline Adrenal (right) & $0.031 \pm 0.004$ & $0.033 \pm 0.006$ & $0.035 \pm 0.007$ & $0.034 \pm 0.005$ \\
\hline$\%$ to bodyweight & $0.012 \pm 0.002$ & $0.012 \pm 0.002$ & $0.012 \pm 0.002$ & $0.013 \pm 0.002$ \\
\hline Kidney (left) & $0.830 \pm 0.076$ & $0.877 \pm 0.078$ & $0.920 \pm 0.150$ & $0.890 \pm 0.092$ \\
\hline$\%$ to bodyweight & $0.324 \pm 0.038$ & $0.322 \pm 0.037$ & $0.315 \pm 0.028$ & $0.329 \pm 0.032$ \\
\hline Kidney (right) & $0.854 \pm 0.067$ & $0.885 \pm 0.072$ & $0.938 \pm 0.151$ & $0.915 \pm 0.095$ \\
\hline$\%$ to bodyweight & $0.333 \pm 0.032$ & $0.325 \pm 0.033$ & $0.321 \pm 0.028$ & $0.338 \pm 0.029$ \\
\hline Liver & $6.476 \pm 0.617$ & $7.097 \pm 1.090$ & $7.528 \pm 1.188$ & $7.196 \pm 0.918$ \\
\hline$\%$ to bodyweight & $2.516 \pm 0.189$ & $2.586 \pm 0.275$ & $2.572 \pm 0.168$ & $2.651 \pm 0.202$ \\
\hline Ovary (left) & $0.039 \pm 0.015$ & $0.036 \pm 0.012$ & $0.036 \pm 0.009$ & $0.036 \pm 0.005$ \\
\hline$\%$ to bodyweight & $0.015 \pm 0.006$ & $0.013 \pm 0.004$ & $0.012 \pm 0.003$ & $0.013 \pm 0.003$ \\
\hline Ovary (right) & $0.038 \pm 0.016$ & $0.038 \pm 0.014$ & $0.033 \pm 0.010$ & $0.07 \pm 0.010$ \\
\hline$\%$ to bodyweight & $0.015 \pm 0.006$ & $0.014 \pm 0.005$ & $0.011 \pm 0.004$ & $0.014 \pm 0.004$ \\
\hline Uterus and Cervix & $0.649 \pm 0.222$ & $0.700 \pm 0.293$ & $0.722 \pm 0.180$ & $0.569 \pm 0.157$ \\
\hline \% to bodyweight & $0.253 \pm 0.089$ & $0.257 \pm 0.115$ & $0.249 \pm 0.065$ & $0.208 \pm 0.044$ \\
\hline No. of animals & 10 & 10 & 10 & 10 \\
\hline
\end{tabular}

(a) Bodyweights were measured immediately prior to necropsy after an overnight fast. Significant differences were compared with the control group, ${ }^{*} P<0.05$.

Table 13. Necropsy findings in 13 weeks repeated oral dose toxicity of WG extract in male rats.

\begin{tabular}{|c|c|c|c|c|c|}
\hline \multirow{2}{*}{ Parameters } & \multirow{2}{*}{$\begin{array}{c}\text { Observation } \\
\text { Type }\end{array}$} & \multicolumn{4}{|c|}{ Dose (mg/kg/day) } \\
\hline & & Control & 1250 & 2500 & 5000 \\
\hline \multicolumn{6}{|l|}{ Male } \\
\hline Adrenal Gland & Normal & 10 & 10 & 10 & 10 \\
\hline Aorta & Normal & 10 & 10 & 10 & 10 \\
\hline Bone Marrow, Sternum & Normal & 10 & 10 & 10 & 10 \\
\hline Brain & Normal & 10 & 10 & 10 & 10 \\
\hline Cervix & Normal & - & - & - & - \\
\hline Coagulating Gland & Normal & 10 & 10 & 10 & 10 \\
\hline Epididymis & Normal & 10 & 10 & 10 & 10 \\
\hline Esophagus & Normal & 10 & 10 & 10 & 10 \\
\hline Eye with Optic Nerve & Normal & 10 & 10 & 10 & 10 \\
\hline Harderian Gland & Normal & 10 & 10 & 10 & 10 \\
\hline Heart & Normal & 10 & 10 & 10 & 10 \\
\hline Cecum & Normal & 10 & 10 & 10 & 10 \\
\hline Colon & Normal & 10 & 10 & 10 & 10 \\
\hline Duodenum & Normal & 10 & 10 & 10 & 10 \\
\hline Ileum & Normal & 10 & 10 & 10 & 10 \\
\hline Jejunum & Normal & 10 & 10 & 10 & 10 \\
\hline Rectum & Normal & 10 & 10 & 10 & 10 \\
\hline Kidney & Normal & 10 & 10 & 10 & 10 \\
\hline
\end{tabular}


Table 13. Cont.

\begin{tabular}{|c|c|c|c|c|c|}
\hline \multirow{2}{*}{ Parameters } & \multirow{2}{*}{$\begin{array}{c}\text { Observation } \\
\text { Type }\end{array}$} & \multicolumn{4}{|c|}{ Dose (mg/kg/day) } \\
\hline & & Control & 1250 & 2500 & 5000 \\
\hline \multirow[t]{2}{*}{ Liver } & Normal & 10 & 10 & 10 & 10 \\
\hline & Discoloration & 0 & 0 & 0 & 0 \\
\hline Lung & Normal & 10 & 10 & 10 & 10 \\
\hline Lymph Node, Mesenteric & Normal & 10 & 10 & 10 & 10 \\
\hline Lymph Node, Mandibular & Normal & 10 & 10 & 10 & 10 \\
\hline Skeletal Muscle & Normal & 10 & 10 & 10 & 10 \\
\hline Nerve, Peripheral & Normal & 10 & 10 & 10 & 10 \\
\hline Ovary & Normal & - & - & - & - \\
\hline Pancreas & Normal & 10 & 10 & 10 & 10 \\
\hline \multirow[t]{2}{*}{ Parathyroid Gland } & Normal & 9 & 10 & 10 & 10 \\
\hline & Absent & 1 & 0 & 0 & 0 \\
\hline Pituitary Gland & Normal & 10 & 10 & 10 & 10 \\
\hline Prostate Gland & Normal & 10 & 10 & 10 & 10 \\
\hline Salivary Gland & Normal & 10 & 10 & 10 & 10 \\
\hline Seminal Vesicle & Normal & 10 & 10 & 10 & 10 \\
\hline \multirow[t]{3}{*}{ Skin } & Normal & 10 & 10 & 10 & 9 \\
\hline & Alopecia & 0 & 0 & 0 & 1 \\
\hline & Crust & 0 & 0 & 0 & 1 \\
\hline Skin, Mammary & Normal & 10 & 10 & 10 & 10 \\
\hline Spinal Cord, Thoracic & Normal & 10 & 10 & 10 & 10 \\
\hline Spleen & Normal & 10 & 10 & 10 & 10 \\
\hline Stomach & Normal & 10 & 10 & 10 & 10 \\
\hline Testis & Normal & 10 & 10 & 10 & 10 \\
\hline Thymus & Normal & 10 & 10 & 10 & 10 \\
\hline \multirow[t]{3}{*}{ Thyroid Gland } & Normal & 9 & 10 & 10 & 10 \\
\hline & Enlarged & 1 & 0 & 0 & 0 \\
\hline & Absent & 1 & 0 & 0 & 0 \\
\hline Tongue & Normal & 10 & 10 & 10 & 10 \\
\hline Trachea & Normal & 10 & 10 & 10 & 10 \\
\hline Urinary Bladder & Normal & 10 & 10 & 10 & 10 \\
\hline Uterus & Normal & - & - & - & - \\
\hline Vagina & Normal & - & - & - & - \\
\hline Femorotibial Joint & Normal & 10 & 10 & 10 & 10 \\
\hline No. of animals & & 10 & 10 & 10 & 10 \\
\hline
\end{tabular}

-: Not applicable.

Table 14. Necropsy findings in 13 weeks repeated oral dose toxicity of WG extract in female rats.

\begin{tabular}{cccccc}
\hline \multirow{2}{*}{ Parameters } & Observation & \multicolumn{4}{c}{ Dose $\mathbf{( m g} / \mathbf{k g} /$ day) } \\
\cline { 3 - 5 } & Type & Control & $\mathbf{1 2 5 0}$ & $\mathbf{2 5 0 0}$ & $\mathbf{5 0 0 0}$ \\
\hline Female & & & & & \\
Adrenal Gland & Normal & 10 & 10 & 10 & 10 \\
Aorta & Normal & 10 & 10 & 10 & 10 \\
Bone Marrow, Sternum & Normal & 10 & 10 & 10 & 10 \\
Brain & Normal & 10 & 10 & 10 & 10 \\
Coagulating Gland & Normal & - & - & - & - \\
Epididymis & Normal & - & - & - & - \\
Esophagus & Normal & 10 & 10 & 10 & 10 \\
Eye with Optic Nerve & Normal & 10 & 10 & 10 & 10 \\
Harderian Gland & Normal & 10 & 10 & 10 & 10 \\
Heart & Normal & 10 & 10 & 10 & 10 \\
Cecum & Normal & 10 & 10 & 10 & 10 \\
Colon & Normal & 10 & 10 & 10 & 10 \\
\hline
\end{tabular}


Table 14. Cont.

\begin{tabular}{|c|c|c|c|c|c|}
\hline \multirow{2}{*}{ Parameters } & \multirow{2}{*}{$\begin{array}{c}\text { Observation } \\
\text { Type }\end{array}$} & \multicolumn{4}{|c|}{ Dose (mg/kg/day) } \\
\hline & & Control & 1250 & 2500 & 5000 \\
\hline Duodenum & Normal & 10 & 10 & 10 & 10 \\
\hline Ileum & Normal & 10 & 10 & 10 & 10 \\
\hline Jejunum & Normal & 10 & 10 & 10 & 10 \\
\hline Rectum & Normal & 10 & 10 & 10 & 10 \\
\hline Kidney & Normal & 10 & 10 & 10 & 10 \\
\hline \multirow{2}{*}{ Liver } & Normal & 10 & 9 & 10 & 10 \\
\hline & Discoloration & 10 & 1 & 10 & 10 \\
\hline Lung & Normal & 10 & 10 & 10 & 10 \\
\hline Lymph Node, Mesenteric & Normal & 10 & 10 & 10 & 10 \\
\hline Lymph Node, Mandibular & Normal & 10 & 10 & 10 & 10 \\
\hline Skeletal Muscle & Normal & 10 & 10 & 10 & 10 \\
\hline Nerve, Peripheral & Normal & 10 & 10 & 10 & 10 \\
\hline Ovary & Normal & 10 & 10 & 10 & 10 \\
\hline Pancreas & Normal & 10 & 10 & 10 & 10 \\
\hline \multirow[t]{2}{*}{ Parathyroid Gland } & Normal & 10 & 10 & 10 & 10 \\
\hline & Absent & 0 & 0 & 0 & 0 \\
\hline Pituitary Gland & Normal & 10 & 10 & 10 & 10 \\
\hline Prostate Gland & Normal & - & - & - & - \\
\hline Salivary Gland & Normal & 10 & 10 & 10 & 10 \\
\hline Seminal Vesicle & Normal & - & - & - & - \\
\hline \multirow[t]{3}{*}{ Skin } & Normal & 10 & 10 & 10 & 10 \\
\hline & Alopecia & 0 & 0 & 0 & 0 \\
\hline & Crust & 0 & 0 & 0 & 0 \\
\hline Skin, Mammary & Normal & 10 & 10 & 10 & 10 \\
\hline Spinal Cord, Thoracic & Normal & 10 & 10 & 10 & 10 \\
\hline Spleen & Normal & 10 & 10 & 10 & 10 \\
\hline Stomach & Normal & 10 & 10 & 10 & 10 \\
\hline Testis & Normal & - & - & - & - \\
\hline Thymus & Normal & 10 & 10 & 10 & 10 \\
\hline \multirow[t]{3}{*}{ Thyroid Gland } & Normal & 10 & 10 & 10 & 10 \\
\hline & Enlarged & 0 & 0 & 0 & 0 \\
\hline & Absent & 0 & 0 & 0 & 0 \\
\hline Tongue & Normal & 10 & 10 & 10 & 10 \\
\hline Trachea & Normal & 10 & 10 & 10 & 10 \\
\hline Urinary Bladder & Normal & 10 & 10 & 10 & 10 \\
\hline Vagina & Normal & 10 & 10 & 10 & 10 \\
\hline Femorotibial Joint & Normal & 10 & 10 & 10 & 10 \\
\hline \multirow{2}{*}{ Uterus and Cervix } & Normal & 9 & 7 & 8 & 9 \\
\hline & Retention & 1 & 3 & 2 & 1 \\
\hline No. of animals & & 10 & 10 & 10 & 10 \\
\hline
\end{tabular}

-: Not applicable.

\subsubsection{Histopathology}

Histopathological examination was conducted in the control group and the highest dose group (5000 mg/ $\mathrm{kg} /$ day), and organs with abnormal findings the identified in necropsy examination (data not shown). Luminal dilation of the uterus with retention of clear fluid was observed in one female rat in the control group, three female rats of the $1250 \mathrm{mg} / \mathrm{kg}$ /day group, two female of the $2500 \mathrm{mg} / \mathrm{kg} /$ day group, and one female rat of the $5000 \mathrm{mg} / \mathrm{kg} /$ day group, respectively. In addition, necrosis lesions in the liver with discoloration were observed in one female rat of the $1250 \mathrm{mg} / \mathrm{kg}$ /day group.

\section{Discussion}

In this study, the potential toxicity of the WG extract was evaluated by a single oral dose toxicity study, and by 4 -week and 13-week repeated oral dose toxicity studies using Sprague-Dawley rats. The information about the safety of the WG extract obtained 
through the general toxicity study, including gross toxicity, NOAEL, and target organs, will be contributed to registering the Glycyrrhiza new variety (Wongam) in the Korean Pharmacopoeia of the Ministry of Food and Drug Safety.

In the single oral dose toxicity study, oral administration of WG extract did not cause mortality or acute toxicity in rats, and we suggest that the ALD of WG extract is higher than $5000 \mathrm{mg} / \mathrm{kg}$. Compound-colored stool was observed in both sexes of the 2500 and $5000 \mathrm{mg} / \mathrm{kg}$ groups, which was assumed to result from tinting of the stool, due to the color of WG or its excreted metabolites. However, this finding was not considered to be an adverse effect, since it occurred only transiently and ultimately disappeared, with no other abnormal findings in bodyweight or gross pathology at necropsy (Tables 1-3).

In the 4-week repeated oral dose toxicity study, no treatment-related adverse effects were observed involving mortality, clinical signs, ophthalmological examination, bodyweight, food and water consumption, urinalysis, hematology, serum biochemistry, absolute and relative organ weights, or necropsy, at doses up to $5000 \mathrm{mg} / \mathrm{kg} /$ day. No deaths or ophthalmologic abnormalities were observed in either sex (Table S1). As in the single oral dose toxicity study, compound-colored stool was observed in both sexes of the $1250 \mathrm{mg} / \mathrm{kg} /$ day and higher dose groups, attributable to the color of WG or its excreted metabolites. Although the loss of fur was observed in one male rat in the $1250 \mathrm{mg} / \mathrm{kg} /$ day group, this was not considered as related to administering the WG extract owing to the very low incidence rate and the absence of a clear relationship between dose and response. Moreover, loss of fur occurring spontaneously following systemic administration has been reported in previous toxicity studies [16]. Salivation was observed in three male rats of the $5000 \mathrm{mg} / \mathrm{kg} /$ day group, which was considered as related to administering the WG extract because it occurred in the highest dose group. However, it was considered to be a temporary physiological reaction resulting from features of WG extract, such as taste and smell.

Bodyweight was not significantly changed in the treatment groups compared to the control group during the 4-week repeated oral dose toxicity study (Figure S1). Although food consumption significantly increased in the 625 and $5000 \mathrm{mg} / \mathrm{kg} /$ day groups, and significantly decreased in female rats of the 2500 and $5000 \mathrm{mg} / \mathrm{kg} /$ day groups, this was not attributed to administering the WG extract, since it occurred transiently and was not accompanied by any corresponding weight changes (Figure S2). Similarly, the significantly increased water consumption of female rats in all dose groups, and the significantly decreased water consumption of male rats of the 625 and $1250 \mathrm{mg} / \mathrm{kg} /$ day groups and female rats of the 625,1250 , and $2500 \mathrm{mg} / \mathrm{kg} /$ day groups were not considered to be related to the WG extract, since the responses were sporadic and not dose-related, with no corresponding changes in bodyweight or serum biochemistry (Figure S3). Furthermore, these changes in food and water consumption remained within the normal range of the reference data [17].

During the 4-week repeated oral dose toxicity study, no significant changes were observed in urinalysis (Tables S2 and S3), hematology (Tables S4 and S5), serum biochemistry (Tables S6 and S7), or organ weights (Tables S8 and S9) in any of the WG-extract dose groups and in rats of either sex compared to the control group.

Hydronephrosis in the right kidney of one male rat treated with $625 \mathrm{mg} / \mathrm{kg} /$ day group was found at necropsy (Tables S10 and S11) during the 4-week repeated oral dose toxicity study. However, this was not considered to be toxicologically significant owing to the very low incidence rate and the absence of any correlation with dose-dependent responses. Moreover, the response was not attributable to the WG extract in this study, since it has been reported as occurring spontaneously in Sprague-Dawley rats in previous studies $[18,19]$. A histopathological examination was not performed, as there were no organs with significant gross lesions.

In the 13-week repeated oral dose toxicity study, no death or ophthalmologic abnormalities were observed in rats of either sex at doses up to $5000 \mathrm{mg} / \mathrm{kg} /$ day. Similarly to the single oral dose toxicity study and the 4-week repeated oral dose toxicity study, 
compound-colored stool and salivation were observed in all dose groups in both sexes and in eight male rats of the $5000 \mathrm{mg} / \mathrm{kg} /$ day group, respectively. Loss of fur and wound scratching were observed in two male rats of the $5000 \mathrm{mg} / \mathrm{kg} /$ day group, and crusted skin formation was observed in one of these (Table 4). These signs were not considered as related to administering the WG extract for the same reasons as with the 4-week repeated oral dose toxicity study.

No significant changes in bodyweight or food consumption were observed in male rats in relation to administering the WG extracts. By contrast, bodyweight was significantly increased during most experiment periods involving female rats of the $2500 \mathrm{mg} / \mathrm{kg} /$ day group, while food consumption was significantly increased at week 3 in female rats of the $2500 \mathrm{mg} / \mathrm{kg}$ /day group. These increases were not considered as related to administering the WG extract, since they were not dose-related (Figures 1 and 2). Water consumption did not significantly change after administration WG extract in any of the dose groups of either sex compared to the control group (Figure 3).

In the urinalysis tests, the ketone body was increased in both sexes of the $5000 \mathrm{mg} / \mathrm{kg} /$ day group. Specific gravity was increased in male rats of the 2500 and $5000 \mathrm{mg} / \mathrm{kg} /$ day groups (Tables 5 and 6). These changes were related to administering the WG extract because the responses were dose-dependent and accompanied by increasing kidney weights. However, they were not considered to be toxicologically significant, since they remained within the normal range of the reference data and were not accompanied by histopathological changes in other related items [17].

In the hematology tests, RET tended to decrease in male rats of the 2500 and $5000 \mathrm{mg} / \mathrm{kg} /$ day group (Table 7). However, this was not considered as related to administering the WG extracts, since the RET ratio of the control group in this study was higher than the normal levels in other reports with the same conditions [20-24].

In the serum biochemistry tests, Na was significantly increased in male rats of the 2500 and $5000 \mathrm{mg} / \mathrm{kg} /$ day group (Table 9). ALP was significantly increased in female rats of the $5000 \mathrm{mg} / \mathrm{kg} /$ day group (Table 10). These changes were considered as related to administering the WG extract, since the responses were dose-dependent. However, they were not considered to be toxicologically significant, since they remained within the normal range of the reference data and were not accompanied by histopathological changes in other related items [17].

The relative organ weight of the left kidney and the absolute organ weight of the right testis were significantly increased in male rats of the $5000 \mathrm{mg} / \mathrm{kg} /$ day and $1250 \mathrm{mg} / \mathrm{kg} /$ day groups, respectively (Table 11). In addition, the absolute organ weight of the lung was significantly increased in female rats of the 1250 and $2500 \mathrm{mg} / \mathrm{kg} /$ day groups (Table 12). These changes were not considered as related to administering the WG extract, since the responses were not dose-related.

In the necropsy findings (Tables 13 and 14) and histopathological tests (data not shown), the absence of the left parathyroid gland and the left thyroid gland, and enlargement of the right thyroid gland were observed in one male rat in the control group. Alopecia and skin crusting were observed in one male rat of the $5000 \mathrm{mg} / \mathrm{kg} /$ day group through necropsy and histopathology tests. Retention of clear fluid in the uterus in the necropsy findings was caused by luminal dilation, observed in histopathological tests. Discoloration of the liver, observed in necropsy findings, was caused by focal/multifocal necrosis, which was also observed in histopathological tests. These changes were judged to be neither significant nor dose-related, having occurred spontaneously in Sprague-Dawley rats [25]. Furthermore, necropsy findings did not correlate with histopathology.

Interestingly, chronic ingestion of licorice or licorice-like compounds (such as carbenoxolone) could lead to the temporary form of apparent mineralocorticoid excess (AME), which is an autosomal recessive disorder. AME is characterized by hypertension, hypokalemia, metabolic alkalosis, and low plasma renin activity, and aldosterone level [26]. The major etiology of AME is mutations in the 11 $\beta$-hydroxysteroid dehydrogenase type $2(11 \beta-H S D 2)$ gene, which catalyzes the conversion of active cortisol to inactive cortisol 
in the kidney. When a $11 \beta$-HSD2 mutation occurrs, it causes high concentrations of cortisol in the kidney. Then it can cross-react and activate the mineralocorticoid receptor, which leads to the AME-associated syndromes $[27,28]$. It is noteworthy that glycyrrhetinic acid, which is the hydrolysis of glycyrrhizic acid, has been identified as an inhibitor of $11 \beta$-HSD2 [26]. Therefore, long term ingestion of licorice could occur the possibility of AME. In our results, there are no abnormal signs associated with AME in the single oral dose and 4-week repeated oral dose toxicity study. However, a significantly increase of the urinary ketone body and protein (Tables 5 and 6), serum Na (Table 9), and kidney weight (Table 11) occurred in a 13-week repeated oral dose toxicity study. Although we considered that these changes were not toxicologically significant, due to the within the normal range of the reference data and not accompanied by histopathological changes, it seemed to be a possibility of AME development. Besides, WG has higher levels of glycyrrhizic acid than other species of licorice. Additionally, previous studies reported that AME-associated symptoms are not present with consumption of less than $50 \mathrm{~g} /$ day of licorice, although several practical difficulties [29]. Human equivalent dose (HED) upon the $5000 \mathrm{mg} / \mathrm{kg}$ of WG extracts in rats is almost $811 \mathrm{mg} / \mathrm{kg}$ [30]. Therefore, we suggest that long term treatment of WG high dose should be carefully given to patients, due to the possibility of AME.

Toxicological effects of the Glycyrrhiza radix have been reported in many previous studies. In a single oral dose toxicity study of G. glabra in rats, ethanol and aqueous extracts did not cause mortality at up to $1000 \mathrm{mg} / \mathrm{kg}$ over 14 days. In the administration of the $2000 \mathrm{mg} / \mathrm{kg}$ of these extracts, slight gross behavioral changes were observed, including alertness, spontaneous locomotor activity, and reactivity to touch [31]. A 15-day repeated oral dose toxicity of G. glabra, aqueous extract (100, 250, and $500 \mathrm{mg} / \mathrm{kg} /$ day) in rats is reported in one study. Suppression of the adrenal-pituitary axis, decreased plasma concentrations of cortisol, adrenocorticotropic hormone (ACTH), aldosterone, and $\mathrm{K}+$, and increased plasma concentrations of renin and $\mathrm{Na}+$ were observed in a dose-dependent manner [32]. In addition, a 9-week repeated oral dose toxicity study of water extract of G. uralensis was conducted in rats. The NOAEL of water extract of G. uralensis was reported to be higher than $2000 \mathrm{mg} / \mathrm{kg} /$ day in male rats, despite a slight decrease in prostate weight and daily sperm production [33]. A 13-week repeated oral dose toxicity study was conducted with flavonoid oil of G. glabra instead of water extract. The NOAEL of flavonoid oil of G. glabra was estimated to be $400 \mathrm{mg} / \mathrm{kg} /$ day in male rats, and $800 \mathrm{mg} / \mathrm{kg} /$ day in female rats [34]. Although we only estimated the general toxicity of WG in this study, and not the toxicity of other Glycyrrhiza species, we could infer that the toxicity of other Glycyrrhiza species is higher than that of the Glycyrrhiza new variety (Wongam) through comparison with the previous reports.

In conclusion, we report that the oral ALD and NOAEL of WG are higher than $5000 \mathrm{mg} / \mathrm{kg}$ in rats of both sexes, and that no target organs were identified. We accordingly assume that WG is of lower general toxicity than that of other Glycyrrhiza species reported in previous studies. The results of the present study are thus expected to contribute to the registration of the Glycyrrhiza new variety (Wongam) in the Korean Pharmacopoeia.

\section{Materials and Methods}

\subsection{Wongam Preparation}

WG was obtained from the Korean Rural Development Administration, and extracted by Wonkwang Herb Co. (Jinan-gun, Jeollabuk-do, Republic of Korea). Briefly, WG was extracted with distilled water at $100^{\circ} \mathrm{C}$ for $4 \mathrm{~h} 30 \mathrm{~min}$. The extract was concentrated under reduced pressure in a rotary evaporator at $70{ }^{\circ} \mathrm{C}$ for $3 \mathrm{~h}$. The decoction was filtered using Whatman filter paper no.1. Then, it was lyophilized (Batch methods) and stored at $4{ }^{\circ} \mathrm{C}$. The yield of the dried extract from the starting crude of the WG was $8.8 \%$. The WG extract was prepared for administration by suspending with sterile water for injection according to the doses assigned for each group in the single and repeated oral dose toxicity studies. 


\subsection{Experimental Animals and Animal Husbandry}

All toxicological studies were carried out by Chemon Inc. under Good Laboratory Practice (GLP) conditions. Specific pathogen-free Sprague-Dawley rats were obtained from Orient bio Inc. (Gapyeong, Gyeonggi-do, Republic of Korea), and used for the single and repeated oral dose toxicity studies. The studies were approved by the Institutional Animal Care and Use Committee (IACUC) of the Preclinical Research Center, Chemon Inc. (Approval Number: 19-R491 for the single oral dose toxicity study and 19-R654 for the 13-week repeated oral dose toxicity study). Animals were housed in the laboratory animal facility at a temperature of $23 \pm 3^{\circ} \mathrm{C}$ and relative humidity of $55 \pm 15 \%$. Animal housing was maintained under a 12-h light-dark cycle, with 10-20 air changes per hour. Animals were supplied irradiation-sterilized pellet feed (Teklad Certified Irradiated Global 18\% Protein Rodent Diet, 2918C; Envigo RMS, Inc., IN, USA.), along with tap water disinfected using an ultraviolet sterilizer and ultrafiltration ad libitum. All animals were acclimated for 6 days before the start of the experiment. The study was conducted in accordance with test guidelines from the Korean Ministry of Food and Drug Safety (MFDS, 2018) and guidelines for the testing of chemicals from the Organization for Economic Cooperation and Development (OECD, 1997) under GLP Regulations.

\subsection{Single Oral Dose Toxicity Study}

8-week-old Sprague-Dawley male and female rats ( $\mathrm{n}=5$ per sex and group) were orally treated with WG at 0 (Control), 1250, 2500, and $5000 \mathrm{mg} / \mathrm{kg}$. Animals were housed in stainless steel cages with mesh flooring. No more than three animals were housed per cage during the quarantine and acclimation period, and the animals were housed individually during the dosing and observation periods. Clinical signs and mortality were monitored constantly for the first $30 \mathrm{~min}$ after dose administration, then every hour until $6 \mathrm{~h}$ after oral treatment, and daily for 14 days subsequently. During the 15-day experimental period, the bodyweight of all rat groups was recorded. Then, all animals were euthanized by inhaled carbon dioxide (CO2) on Day 15, and gross findings were observed at necropsy.

\subsection{3-Week Repeated Oral Dose Toxicity Study}

The high dose was set at $5000 \mathrm{mg} / \mathrm{kg} /$ day, based on the absence of toxic signs in a 4-week repeated oral dose toxicity study (Figures S1-S3 and Tables S1-S11). 6-week-old male and female Sprague-Dawley rats ( $\mathrm{n}=10$ per sex and group) were orally administered WG at 0 (control), 1250, 2500, and $5000 \mathrm{mg} / \mathrm{kg} /$ day for 13 weeks. Bodyweight ranges at the initiation of dosing were 207.93-235.53 $\mathrm{g}$ for males and 136.04-165.50 $\mathrm{g}$ for females, respectively.

Animals were individually checked once a day to observe any clinical signs and mortalities, and the type, date of occurrence, and severity of signs were recorded. Bodyweights of all rat groups were recorded before the initiation of dosing (Day 1) and once a week during the experimental period. Before necropsy, all rat groups were fasted overnight, and bodyweights were recorded at necropsy. Food and water intake were checked on the same days as bodyweight measurements were recorded. The eyes of five male and five female rats per group were macroscopically evaluated during the final week of observation. A mydriatic (Mydriacyl Eye Drops 1\%, Alcon Korea) was dropped into both eyeballs to facilitate mydriasis, after which the anterior parts of the eye, optic media, and fundus were observed with a Keeler Vantage Plus LED Digital Binocular Ophthalmoscope (Keeler Instruments Inc., PA, USA).

Urinalysis was performed during the last week of observation; 5 male and 5 female rats per group were individually housed in a stainless-steel cage cleaned and disinfected with $70 \%$ alcohol. Urine samples were collected, and $0.3 \mathrm{~mL}$ of fresh urine was taken for analysis. Urine samples were analyzed for glucose, bilirubin, ketone body, specific gravity, $\mathrm{pH}$, protein, urobilinogen, nitrite, occult blood, red blood cell (RBC), white blood cell (WBC), epithelial cell, urine color, clarity, cast, and total urine volume using an automatic analyzer (Clinitek Advantus; Siemens, Munchen, Germany). 
For necropsy, animals were euthanized by inhalation of 3-5\% isoflurane (Terel liquid; Kyongbo Pharma. Co., Ltd., Asan-si, Chungcheongnam-do, Republic of Korea) on Day 91. Blood samples were collected from the posterior vena cava for hematological and serum biochemical testing. Approximately $1 \mathrm{~mL}$ of blood was placed in a CBC bottle (Vacutainer $3 \mathrm{~mL}$; BD) with anticoagulant EDTA-2K. Hematology parameters were measured using a hematology analyzer (ADVIA 2120; Siemens, Munchen, Germany), including $\mathrm{RBC}$, hemoglobin (HGB), hematocrit (HCT), mean corpuscular volume (MCV), mean corpuscular hemoglobin $(\mathrm{MCH})$, mean corpuscular hemoglobin concentration $(\mathrm{MCHC})$, red cell distribution width (RDW), hemoglobin distribution width (HDW), reticulocyte (RET), platelet count (PLT), mean platelet volume (MPV), white blood cell count (WBC), neutrophils count (NEU), lymphocytes count (LYM), monocytes count (MONO), eosinophils count (EOS), basophils count (BASO), and large unstained cell count (LUC). Activated partial thromboplastin time (APTT) and prothrombin time (PT) were measured using a coagulation analyzer (CS-1600; Sysmex; Kobe, Hyogo, Japan).

Serum biochemical parameters were measured using a serum biochemistry analyzer (AU680; Beckman Coulter; CA, USA). About $2 \mathrm{~mL}$ of the blood sample was added to a $5 \mathrm{~mL}$ Vacutainer tube (SST ${ }^{\mathrm{TM}}$ II Advance; BD; NJ, USA) that contained a clot activator. Blood was coagulated by being left at room temperature for 15-20 min and then centrifuged for $10 \mathrm{~min}$ (3000 rpm, 1902 Relative Centrifugal Force (RCF), Combi-514R; Hanil, Republic of Korea) to collect serum samples. Parameters examined were aspartate aminotransferase (AST), alanine aminotransferase (ALT), alkaline phosphatase (ALP), creatine phosphokinase (CPK), total bilirubin (TBIL), glucose (GLU), total cholesterol (TCHO), triglyceride (TG), total protein (TP), albumin (ALB), albumin/globulin (A/G) ratio, blood urea nitrogen (BUN), creatinine (CREA), inorganic phosphorus (IP), calcium (Ca), sodium (Na), potassium ion $(\mathrm{K})$ and chloride ion $(\mathrm{Cl})$.

After blood sampling, animals were sacrificed by exsanguination from the abdominal aorta and posterior vena cava. Gross findings were recorded, including body surface, subcutis, head, and all organs in the abdominal and thoracic cavities. Following this, organs were weighed using an electronic balance (Sartorius AG): brain, pituitary gland, lung heart, thymus, spleen, adrenal (both), kidney (both), liver, testis (both), epididymis (both), and prostate gland. Organ weights (\%) relative to terminal bodyweights were also calculated.

Histopathological examination was performed on all organs collected from the control and highest dose $(5000 \mathrm{mg} / \mathrm{kg} /$ day) groups, and additionally on macroscopically abnormal organs from the low- and mid-dose groups.

\subsection{Statistical Analysis}

Data are presented as mean \pm standard deviation. Statistical analysis was performed by parametric one-way analysis of variance (ANOVA), assumption of homogeneity was performed by Levene's test, and evaluation of significant differences between the vehicle control and treatment groups was estimated by Dunnett's test using the Provantis ${ }^{\mathrm{TM}} 10.10 .1$ package. The level of significance was taken as $P<0.05$.

In this study, unless specified otherwise, the term "significant" in sentences with P-value implies that inter-group differences have attained statistical significance compared to the control group.

Supplementary Materials: The following are available online at https:/ / www.mdpi.com/article/10 .3390 / plants10061126/s1, Figure S1: Bodyweight of male and female rats in the 4 weeks repeated oral dose toxicity study of WG extract, Figure S2: Food consumption of male and female rats in the 4 weeks repeated oral dose toxicity study of WG extract, Figure S3: Water consumption of male and female rats in the 4 weeks repeated oral dose toxicity study of WG extract, Table S1: Mortality, Clinical signs and Ophthalmological examination in 4 weeks repeated oral dose toxicity of WG, Table S2: Urinalysis in 4 weeks repeated oral dose toxicity of WG in male rats, Table S3: Urinalysis in 4 weeks repeated oral dose toxicity of WG in female rats, Table S4: Hematology values in 4 weeks repeated oral dose toxicity of WG in male rats, Table S5: Hematology values in 4 weeks repeated 
oral dose toxicity of WG in female rats, Table S6: Serum biochemical values in 4 weeks repeated oral dose toxicity of WG in male rats, Table S7: Serum biochemical values in 4 weeks repeated oral dose toxicity of WG in female rats, Table S8: Absolute and relative organ weights in 4 weeks repeated oral dose toxicity of WG in male rats, Table S9: Absolute and relative organ weights in 4 weeks repeated oral dose toxicity of WG in female rats, Table S10: Necropsy findings in 4 weeks repeated oral dose toxicity of WG in male rats, Table S11: Necropsy findings in 4 weeks repeated oral dose toxicity of WG in female rats.

Author Contributions: Conceptualization, Y.-D.J. and J.-S.J.; resources, J.L. and J.C.; data curation, J.L. and D.-G.K.; writing (original draft preparation), D.-G.K.; writing (review and editing), J.L., W.K., H.-J.A., J.-H.L., J.C., S.-H.K., Y.-J.S., Y.-D.J. and J.-S.J.; visualization, D.-G.K. All authors have read and agreed to the published version of the manuscript.

Funding: This study was carried out with the support of the "Cooperative Research Program for Agriculture Science and Technology Development (Project No. PJ01424602)" Rural Development Administration, Republic of Korea.

Institutional Review Board Statement: The study was conducted in accordance with test guidelines from the Korean Ministry of Food and Drug Safety (MFDS, 2018) and guidelines for the testing of chemicals from the Organization for Economic Cooperation and Development (OECD, 1997) under GLP Regulations. The studies were approved by the Institutional Animal Care and Use Committee (IACUC) of the Preclinical Research Center, Chemon Inc. (Approval Number: 19-R491 for the single oral dose toxicity study, 19-R512 for the 4-week repeated oral dose toxicity study, and 19-R654 for the 13-week repeated oral dose toxicity study).

Informed Consent Statement: Not applicable.

Data Availability Statement: Datasets used and/or analyzed in the current study are available from the corresponding author on reasonable request.

Conflicts of Interest: The authors declare no conflict of interest.

\section{References}

1. Liao, W.C.; Lin, Y.-H.; Chang, T.-M.; Huang, W.-Y. Identification of two licorice species, Glycyrrhiza uralensis and Glycyrrhiza glabra, based on separation and identification of their bioactive components. Food Chem. 2012, 132, 2188-2193. [CrossRef]

2. Pastorino, G.; Cornara, L.; Soares, S.; Rodrigues, F.; Oliveira, M.B.P.P. Liquorice (Glycyrrhiza glabra): A phytochemical and pharmacological review. Phytother. Res. 2018, 32, 2323-2339. [CrossRef]

3. Kwon, Y.-J.; Son, D.-H.; Chung, T.-H.; Lee, Y.-J. A Review of the Pharmacological Efficacy and Safety of Licorice Root from Corroborative Clinical Trial Findings. J. Med. Food 2020, 23, 12-20. [CrossRef]

4. Zhang, Q.; Ye, M. Chemical analysis of the Chinese herbal medicine Gan-Cao (licorice). J. Chromatogr. A 2009, 1216, 1954-1969. [CrossRef]

5. Gao, X.; Wang, W.; Wei, S.; Li, W. [Review of pharmacological effects of Glycyrrhiza radix and its bioactive compounds]. Zhongguo Zhong Yao Za Zhi 2009, 34, 2695-2700.

6. Wang, W.; Hu, X.; Zhao, Z.; Liu, P.; Hu, Y.; Zhou, J.; Zhou, D.; Wang, Z.; Guo, D.; Guo, H. Antidepressant-like effects of liquiritin and isoliquiritin from Glycyrrhiza uralensis in the forced swimming test and tail suspension test in mice. Prog. Neuro-Psychopharmacol. Biol. Psychiatry 2008, 32, 1179-1184. [CrossRef]

7. Hajirahimkhan, A.; Simmler, C.; Yuan, Y.; Anderson, J.R.; Chen, S.-N.; Nikolić, D.; Dietz, B.M.; Pauli, G.F.; van Breemen, R.B.; Bolton, J.L. Evaluation of estrogenic activity of licorice species in comparison with hops used in botanicals for menopausal symptoms. PLoS ONE 2013, 8, e67947. [CrossRef] [PubMed]

8. Lee, J.-H.; Oh, M.-W.; Lee, S.-H.; Park, C.-G.; Jeong, J.-T.; Han, J.-W.; Ma, K.-H.; Chang, J.-K. 'Wongam', a Licorice Interspecific Hybrid Cultivar with High Yield. Korean J. Breed. Sci. 2020, 52, 454-459. [CrossRef]

9. Kang, Y.-M.; Kim, W.; Jin, J.-S.; Lee, J.-H.; Chang, J.K.; Lee, J.; An, H.-J. The Comparative Study of Immunomodulatory Effect by Glycyrrhiza New Varieties and Official Compendia. Korean J. Herbol. 2020, 35, 11-19.

10. Kang, Y.-M.; Kim, W.; Jin, J.-S.; Lee, J.-H.; Chang, J.K.; Lee, J.; An, H.-J. The Comparative Study of Anti-allergic Effect by Glycyrrhiza New Varieties and Official Compendia. Korean J. Herbol. 2020, 35, 13-21.

11. Cho, M.J.; Kim, J.H.; Park, C.H.; Lee, A.Y.; Shin, Y.S.; Lee, J.H.; Park, C.G.; Cho, E.J. Comparison of the effect of three licorice varieties on cognitive improvement via an amelioration of neuroinflammation in lipopolysaccharide-induced mice. Nutr. Res. Pract. 2018, 12, 191-198. [CrossRef] [PubMed]

12. Gosselin, R.-D. Guidelines on statistics for researchers using laboratory animals: The essentials. Lab. Anim. 2019, 53, 28-42. [CrossRef] [PubMed] 
13. Buckley, L.A.; Dorato, M.A. High dose selection in general toxicity studies for drug development: A pharmaceutical industry perspective. Regul. Toxicol. Pharmacol. 2009, 54, 301-307. [CrossRef]

14. Sparrow, S.S.; Robinson, S.; Bolam, S.; Bruce, C.; Danks, A.; Everett, D.; Fulcher, S.; Hill, R.E.; Palmer, H.; Scott, E.W.; et al. Opportunities to minimise animal use in pharmaceutical regulatory general toxicology: A cross-company review. Regul. Toxicol. Pharmacol. 2011, 61, 222-229. [CrossRef]

15. Igarashi, T.; Suzuki, H.; Ushida, K.; Matsumoto, M.; Inoue, K.; Kanno, T.; Miwa, Y.; Ishii, T.; Nagase, T.; Katsumata, Y.; et al. Initial hazard assessment of 1,4-dichlorobutane: Genotoxicity tests, 28-day repeated-dose toxicity test, and reproductive/developmental toxicity screening test in rats. Regul. Toxicol. Pharmacol. 2020, 112, 104610. [CrossRef] [PubMed]

16. Greaves, P. Histopathology of Preclinical Toxicity Studies: Interpretation and Relevance in Drug Safety Studies, 4th ed.; Elsevier: New York, NY, USA, 2012.

17. Han, Z.-Z.; Xu, H.-D.; Kim, K.-H.; Ahn, T.-H.; Bae, J.-S.; Lee, J.-Y.; Gil, K.-H.; Lee, J.-Y.; Woo, S.-J.; Yoo, H.-J.; et al. Reference Data of the Main Physiological Parameters in Control Sprague-Dawley Rats from Pre-clinical Toxicity Studies. Lab. Anim. Res. 2010, 6, 153-164. [CrossRef]

18. Arnold, A.C.; Shaltout, H.A.; Gilliam-Davis, S.; Kock, N.D.; Diz, D.I. Autonomic control of the heart is altered in Sprague-Dawley rats with spontaneous hydronephrosis. Am. J. Physiol. Heart Circ. Physiol. 2011, 300, H2206-H2213. [CrossRef]

19. Chandra, M.; Frith, C.H. Non-neoplastic renal lesions in Sprague-Dawley and Fischer-344 rats. Exp. Toxicol. Pathol. 1994, 45, 439-447. [CrossRef]

20. Shin, S.-H.; Koo, K.-H.; Bae, J.-S.; Cha, S.-B.; Kang, I.-S.; Kang, M.-S.; Kim, H.-S.; Heo, H.-S.; Park, M.-S.; Gil, G.-H.; et al. Single and 90-day repeated oral dose toxicity studies of fermented Rhus verniciflua stem bark extract in Sprague-Dawley rats. Food Chem. Toxicol. 2013, 55, 617-626. [CrossRef] [PubMed]

21. Han, Z.-Z.; Koo, K.-H.; Kim, K.-H.; Bae, J.-S.; Shin, S.-H.; Kim, H.-S.; Kim, J.-H.; Heo, H.-S.; Gil, K.-H.; Lee, J.-Y.; et al. Acute and 90-day subchronic toxicity studies of Silk peptide E5K6, in Sprague-Dawley rats. Food Chem. Toxicol. 2011, 49, $2408-2414$. [CrossRef]

22. Cha, S.-B.; Kim, H.-S.; Bae, J.-S.; Song, S.-W.; Lim, J.-O.; Kim, J.-C. A 13-week subchronic toxicity study of a Dioscorea Rhizome water extract in rats. Regul. Toxicol. Pharmacol. 2021, 120, 104844. [CrossRef]

23. Seong, N.-W.; Seo, H.-S.; Kim, J.-H.; Kim, Y.-J.; Kim, E.; Lee, J.-Y.; Ko, J.-W.; Kim, J.-C. A 13-week subchronic toxicity study of an Eriobotrya japonica leaf extract in rats. J. Ethnopharmacol. 2018, 226, 1-10. [CrossRef]

24. Kim, S.Y.; Kwak, K.-W.; Park, E.-S.; Yoon, H.J.; Kim, Y.-S.; Park, K.; Kim, E.; Kim, S.-D. Evaluation of Subchronic Oral Dose Toxicity of Freeze-Dried Skimmed Powder of Zophobas atratus Larvae (frpfdZAL) in Rats. Foods 2020, 9, 995. [CrossRef] [PubMed]

25. Keenan, C.M.; Baker, J.; Bradley, A.; Goodman, D.G.; Harada, T.; Herbert, R.; Kaufmann, W.; Kellner, R.; Mahler, B.; Meseck, E.; et al. International Harmonization of Nomenclature and Diagnostic Criteria (INHAND): Progress to Date and Future Plans. Toxicol. Pathol. 2015, 43, 730-732. [CrossRef]

26. Gallacher, S.D.; Tsokolas, G.; Dimitropoulos, I. Liquorice-induced apparent mineralocorticoid excess presenting in the emergency department. Clin. Med. 2017, 17, 43-45. [CrossRef] [PubMed]

27. Palermo, M.; Quinkler, M.; Stewart, P.M. Apparent mineralocorticoid excess syndrome: An overview. Arq. Bras. Endocrinol. Metabol. 2004, 48, 687-696. [CrossRef] [PubMed]

28. Sontia, B.; Mooney, J.; Gaudet, L.; Touyz, R.M. Pseudohyperaldosteronism, liquorice, and hypertension. J. Clin. Hypertens. 2008, 10, 153-157. [CrossRef] [PubMed]

29. van Uum, S.H. Liquorice and hypertension. Neth. J. Med. 2005, 63, 119-120.

30. Center for Drug Evaluation and Research (Food and Drug Administration, Rockville, Maryland, USA). Guidance for Industry: Estimating the Maximum Safe Starting Dose in Initial Clinical Trials for Therapeutics in Adult Healthy Volunteers. Document. 2005. Available online: https:/ / www.fda.gov/regulatory-information/search-fda-guidance-documents/estimating-maximumsafe-starting-dose-initial-clinical-trials-therapeutics-adult-healthy-volunteers (accessed on 5 May 2021).

31. Chowdhury, B.; Bhattamisra, S.K.; Das, M.C. Anti-convulsant action and amelioration of oxidative stress by Glycyrrhiza glabra root extract in pentylenetetrazole- induced seizure in albino rats. Indian J. Pharmacol. 2013, 45, 40-43. [CrossRef] [PubMed]

32. Al-Qarawi, A.A.; Abdel-Rahman, H.A.; Ali, B.H.; El Mougy, S.A. Liquorice (Glycyrrhiza glabra) and the adrenal-kidney-pituitary axis in rats. Food Chem. Toxicol. 2002, 40, 1525-1527. [CrossRef]

33. Shin, S.; Jang, J.Y.; Choi, B.-I.; Baek, I.-J.; Yon, J.-M.; Hwang, B.Y.; Park, D.; Jeon, J.H.; Nam, S.-Y.; Yun, Y.W.; et al. Licorice extract does not impair the male reproductive function of rats. Exp. Anim. 2008, 57, 11-17. [CrossRef] [PubMed]

34. Nakagawa, K.; Kitano, M.; Kishida, H.; Hidaka, T.; Nabae, K.; Kawabe, M.; Hosoe, K. 90-Day repeated-dose toxicity study of licorice flavonoid oil (LFO) in rats. Food Chem. Toxicol. 2008, 46, 2349-2357. [CrossRef] [PubMed] 